\title{
Experiments on Exhaust Noise of Tightly Integrated Propulsion Systems
}

\author{
James Bridges ${ }^{*}$, Clifford A. Brown ${ }^{\dagger}$, Richard Bozak ${ }^{\ddagger}$ \\ NASA Glenn Research Center, Cleveland, $\mathrm{OH}$
}

\begin{abstract}
A wide-ranging series of tests have been completed that seek to map the effects of installation, including jet by jet interaction effects, on exhaust noise from various nozzles in forward flight. The primary data was far-field acoustic spectral directivity. The goals of the test series were (i) to generate enough data for empirical models of the different effects, and (ii) to provide data for advanced computational noise predictions methods applied to simplified yet realistic configurations. Data is presented that demonstrate several checks on data quality and that provide an overview of trends observed to date. Among the findings presented here: (i) Data was repeatable between jet rigs for single nozzles with and without surfaces to within $\pm 0.5 \mathrm{~dB}$. (ii) The presence of a second jet caused a strong reduction of the summed noise in the plane of the two plumes and an increase over the expected source doubling in most other azimuthal planes. (iii) The impact of the second jet was reduced when the jets were unheated. (iv) The impact of adding a second isolated rectangular jet was relatively independent of the nozzle aspect ratio up to aspect ratio 8:1. (v) Forward flight had similar impact on a high aspect ratio $(8: 1)$ jet as on an axisymmetric jet, except at the peak noise angle where the impact was less. (vi) The effect of adding a second round jet to a tightly integrated nozzle where the nozzle lip was less than a diameter from the surface was very dependent upon the length of the surface downstream of the nozzle. (vii) When the nozzles were rectangular and tightly integrated with the airframe surface the impact of a second jet was very dependent upon how close together the two jets were. This paper serves as an overview of the test; other papers presented in the same conference will give more detailed analysis of the results.
\end{abstract}

\section{Introduction}

Supersonic civilian aircraft will have to be considerably different than conventional aircraft because their shaping is critical to minimizing the boom heard on the ground. One part of this shaping may be to artfully embed the propulsion in the airframe, minimizing the sudden variations in aircraft cross-section or perhaps hiding pressure sources from non-ideally expanded plumes from reaching observers below the vehicle. Such tight integration of the propulsion offers potential noise reduction from the propulsion system, but may also yield additional noise challenges.

Embedded propulsion concepts often entail two geometric features beyond a simple round nozzle: high aspect ratio cross-section and an extended aft deck. The propulsive exhaust can be on the upper or lower side of the aircraft. The aspect ratio can vary significantly, but practical concerns regarding propulsion performance typically drive designs toward smaller aspect ratios unless there are other over-

\footnotetext{
${ }^{*}$ Research Engineer, Acoustics Branch, AIAA Associate Fellow

${ }^{\dagger}$ Research Engineer, Acoustics Branch, AIAA Member

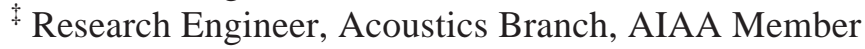


riding objectives that require high aspect ratio. For instance, in the early years of jet propulsion various schemes of augmenting lift by routing exhaust air through slots over airfoils were considered ${ }^{1,2}$. In these studies, aspect ratios up to 100 or more were considered. These exhaust schemes were typically much louder than equivalent isolated round jets, raising concern for current concepts with embedded propulsion.

Research into the causes for the enhanced noise of a jet over a lifting surface has looked at two effects: changes in the turbulent mixing noise, and the addition of a new source from the scattering of turbulent energy as sound. In early studies ${ }^{3,4}$ of the turbulence of a slot jet over a surface it was noted that the turbulence in the shear layer beginning at the trailing edge of the surface was amplified relative to a simple jet shear layer. The degree and scale of the turbulence depended upon the length of the surface downstream of the nozzle exit. This mixing noise source would presumably be included in acoustic analogy approaches, perhaps with a custom Green's function. Having noise generated by scattering at the trailing edge would require a more specialized analysis, such as was first pursued ${ }^{5}$ in 1978 and is being revived ${ }^{6}$.

If there are important gains to be made in aircraft design by having embedded propulsion, the lack of validated tools for predicting noise of turbulent plumes exhausting over surfaces is a significant issue. Most acoustic analogies, using Reynolds-Averaged Navier-Stokes (RANS) computations to provide the flow quantities required, treat only free-space jets, assuming no solid surfaces are near the jet plume. Recent research at NASA aimed at developing a comprehensive database for the impact of solid surfaces on jet noise have shown that strong changes develop in the flow and noise as the surface comes close to the jet plume ${ }^{7}$. Affiliated theoretical development using a rapid distortion theory has attempted to capture this change in terms of a dipole source located at the trailing edge of the surface. This theory is very sensitive to the mean profile and turbulence near the surface and in some ways is not directly applicable to the problem where the jet flow is directly on the surface. Even large eddy simulations (LES) have only recently been applied to this problem ${ }^{8}$, and require validation and guidance as necessary simplifications (particularly regarding the need to resolve the boundary layer on the surface) that must come from experiments. And finally, systems engineers working on vehicle concepts for supersonic flight need simplified, empirical models to make trades among the different performance objectives of the aircraft. For example, the semi-empirical modeling developed by Munro ${ }^{9}$ allows prediction of noise from high aspect ratio jets. In current studies by the NASA High Speed Project aircraft configurations are being studied with low to moderate aspect ratios and with the nozzle exhausting on or near an aft deck, requiring a bit different design space be explored. To this purpose a research program was conceived to systematically extend knowledge of jet noise from single round jets and round jets with parallel surface, to rectangular jets and them with nearby surfaces.

In the Extensible Rectangular Nozzle (ERN11) test, conducted at NASA in 2011, an extensive set of acoustic data was acquired on single-flow convergent rectangular nozzles of aspect ratios 2:1, 4:1, and 8:1 and reported in Ref 10. In addition to variation in aspect ratio, several versions of these nozzles were tested that had an extension of one wide side of the nozzle, characterized as making a 'beveled' nozzle. These nozzles are also representative of exhaust systems with a slot nozzle exiting over an aft deck of the aircraft. One outcome of the test was an empirical model for how the acoustic spectral directivity was affected by the geometry of the nozzle relative to a convergent axisymmetric nozzle. This model, with variables of aspect ratio and aft deck length, consisted of a bilinear polynomial in normalized versions of these variables, and was created to be a model in the new empirical module in NASA's aircraft noise prediction code ANOPP2. This prediction module was limited in the range of aspect ratios and deck lengths that it spanned, and by the initial assumption that the geometric effects on the noise would be independent of jet flow condition. This assumption for the bevel length has since been found to be false, as subsequent work on the impact of jet-surface interactions has shown the dipole nature of some of the noise components. 
For presentation at 20 $0^{\text {th }}$ AIAA/CEAS Aeroacoustics Meeting - 16-18 June 2014

Following the acoustic testing of the ERN nozzles, the ERN12 test was run to measure the flow fields of the rectangular nozzles using PIV. The data from this test was used first to confirm the flow performance that had been predicted (flow uniformity at the nozzle exit), and to document the effect of aspect ratio on the turbulence of the jets. Jet noise prediction codes using RANS input used this data to validate their input.

By 2013 work on the noise impact of round nozzles near surfaces had progressed such that more extensive questions were being asked regarding how having a surface extend far beyond the flowscrubbing part of the nozzle or how having the nozzle standoff from the surface by some amount would change the sound. This became part of the TwinRect/AftDeck test carried out from January -May 2013, described in this paper. Specifically, the first part of the test, which was done on the Small Hot Jet Acoustic Rig (SHJAR) in NASA's Aero-Acoustic Propulsion Lab, focused on single nozzles near surfaces. During this testing the basic rectangular nozzles were again tested; however, the 'bevel' or extended lip of the nozzle was replaced by a plate that extended upstream and to the sides of the nozzle, approximating a semi-infinite surface. The plate could be moved from the lip line outward with a linear actuator, and interchangeable surface extension pieces allowed different lengths of plate beyond the nozzle exit. The length of the plate beyond the nozzle exit was denoted $x_{T E}$ for trailing edge length, and the standoff from the nozzle lip was designated $h$. Two of the new plate configurations replicated the two $x_{T E}$ that had been tested as integral to the rectangular nozzles in the ERN11 test. This configuration could only be acoustically measured in the $0^{\circ}$ and $180^{\circ}$ azimuthal planes (called 'reflected' and 'shielded' respectively in previous jet-surface interaction testing). Geometrically, the range of aft deck lengths covered by the original model was relatively small, extending only up to $x_{T E} / D_{e}=1.25$. In the current work the model has been extrapolated in $x_{T E} / D_{e}$ using the separate plate hardware, up to aft deck lengths of $x_{T E} / D_{e}=6$ for a more complete parametric range of aft deck extensions. Direct comparison of the differences between the two methods of representing the aft deck of aircraft was presented in Ref 11 .

Another precursor test was the twin-jet (TWN10) test performed in the spring of 2010 and documented in Ref 14. These experiments found that the acoustic radiation from twin jets is not simply the addition of $3 \mathrm{~dB}$ to the noise from a single jet. The previous testing showed a variation of up to $6 \mathrm{~dB}$ in the noise emitted to different azimuthal angles. The previous experiments have also shown that the twin jet effects are sensitive to forward flight.

The combined purpose of the TwinRect/AftDeck test being presented in this paper was to expand the effects from twin round jets and surfaces to include the impact of twin rectangular jets and a nearby surface. This test focused on convergent round and rectangular nozzles to concentrate on the effects on the civilian airport takeoff noise of supersonic vehicles proposed by the High Speed Project with highly variable cycles. The TwinRect/AftDeck test also included particle image velocimetry measurements of some configurations, but these results will be covered in another report in the near future.

\section{Test Objectives}

In its essence this test is a seven-variable design test. It is worth noting that if a simple all-combination test design were pursued the test would require 960 configurations and if the minimal flow condition matrix planned here were to be used the test in a full-factorial test design would require over 44,000 points and take almost 3 years of daily testing. Subspaces of the full configuration matrix were created when decimating the test matrix, along with considerable insight on subspace behavior from previous tests, to arrive at a manageable test plan.

Figure 1 was created to assist in understanding the scope of the test. The primary geometric parameters of an installed propulsion system for exhaust noise are the nozzle geometry, the proximity of the nearby surfaces to the jet plume, and the separation of the exhaust plumes from each other. These make up the axes of a design space in the figure. In the lower left-hand corner is the isolated, round nozzle, for which noise prediction methods are available. Some work, including recent testing cited above 
at our facilities, have explored one of the geometric factors singularly, varying the aspect ratio of a rectangular nozzle, looking at plume interaction effects, or the acoustic impact of a nearby surface. But to be able to predict the noise of a highly embedded exhaust system such as are being proposed in recent NASA programs all these effects must be combined. Now, it is too much to expect that the design space will be totally orthogonal, e.g. the effects of each parameter are independent. But the space can be treated as such in experiments to determine the degree of independence and the likely error in using such simple models. At the very least test cases run will serve to validate physics-based prediction codes that do not make such simplifying assumptions.

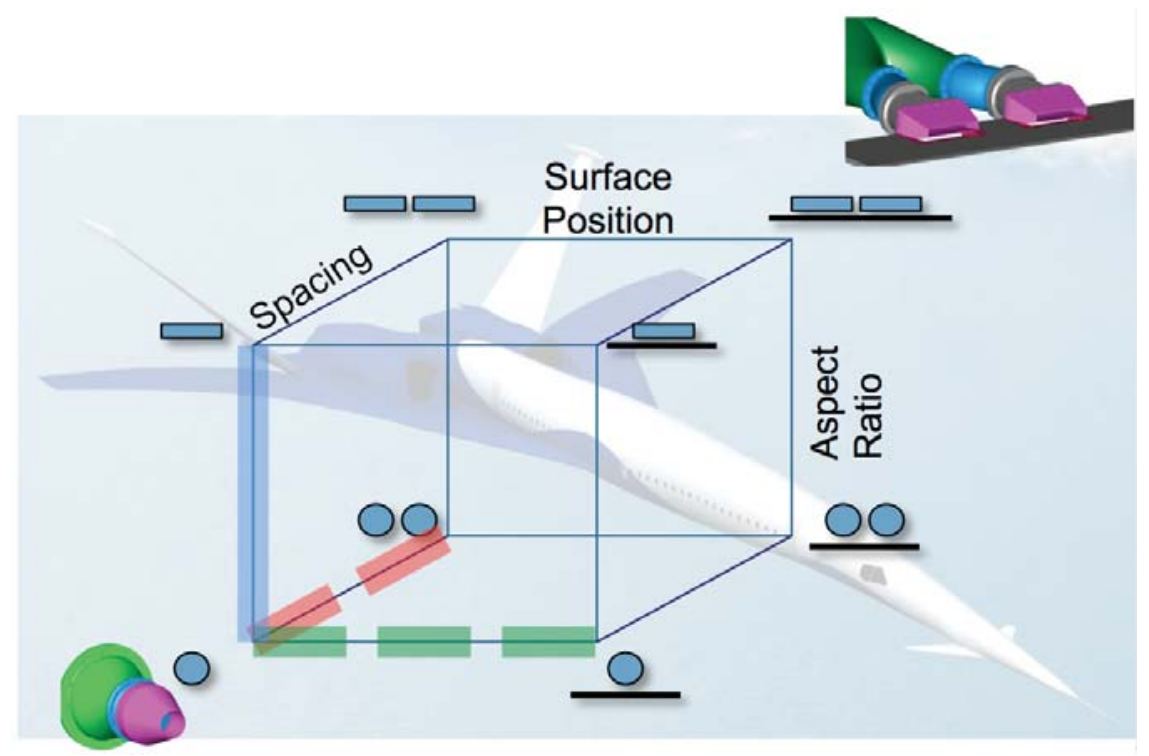

Figure 1 Schematic of parameter space explored in tests described herein.

With these goals and caveats in mind, the present paper will be organized on the following set of objectives:

Objective 1: Document differences in rigs with single jet.

Objective 2: Document the sensitivity to surface span on the jet-surface interaction effect.

Objective 3: Confirm that the acoustic impact of a surface near a single jet is the same in both rigs.

Objective 4: Determine the impact of jet aspect ratio on the acoustic impact of a surface near a single jet.

Objective 5: Determine the impact of jet aspect ratio on the forward flight effect.

Objective 6: Confirm impact of jet spacing on the acoustic spectral directivity from twin round jets.

Objective 7: Determine the impact of jet aspect ratio on the acoustic spectral directivity from twin rectangular jets.

Objective 8: Determine the impact of nearby surface on the acoustic spectral directivity from twin round jets.

Objective 9: Determine the impact of nearby surface on the acoustic spectral directivity from twin rectangular jets.

\section{Experimental Facilities}

The tests were conducted in the NASA Glenn Research Center's Aero-Acoustic Propulsion Laboratory (AAPL). The AAPL is a 65-foot radius anechoic geodesic hemispherical dome. Acoustic wedges cover the inner surfaces of the dome and approximately half of the floor area. AAPL was in its anechoic mode for all acoustic test runs, with acoustic wedges on the front and sides of all rigs and on the floor of the facility. The ambient temperature, pressure, and relative humidity were recorded within the dome, both at the rig centerline and at the top of the dome to check for temperature gradients. 
Two jet rigs are located within AAPL, the Small Hot Jet Acoustic Rig (SHJAR) and the High Flow Jet Exit Rig (HFJER). For economy much of the testing with single jets and surfaces was conducted on the SHJAR, a smaller, single-flow rig without simulated forward flight. A full description of the SHJAR can be found in Ref 12. The rig architecture allowed construction of an articulated surface that more closely met the ideal semi-infinite surface and has been used extensively in past tests of jet-surface interaction effects and of rectangular nozzles ${ }^{13}$. However, SHJAR did not have enough flow capacity to support a second nozzle of reasonable scale (all test were done with a $50.8 \mathrm{~mm}$ or 2" diameter nozzle), nor did it simulate flight.

To extend testing for the effects of a second jet and the effect of flight, a Y-duct adaptor was mounted on the High Flow Jet Exit Rig (HFJER) in the Nozzle Acoustic Test Rig (NATR). The HFJER provides enough hot air flow for two nozzles of 2" diameter and the NATR provides the surrounding flight flow. Details of the HFJER/NATR rigs can be found in Ref 14. The Y-duct adapter attached to the core stream charging station as shown in Figure 2. One difference in the rig from the aforementioned reference was a special fan nozzle constructed to bring the secondary stream duct to a fine lip where the secondary airstream could be made to operate at conditions matching those of the free jet with no wake from the secondary duct.

In both rigs, total and static pressure in the rigs, along with ambient temperature and pressure were recorded with the facility computer, the acoustic data acquisition system being tied to the facility acquisition system to assure accurate documentation of jet plenum conditions during acoustic data acquisition. Total pressure probes in the two legs of the Y-duct adapter confirmed that the Y-duct was producing equal flow in both legs. Acoustic Mach number $M a=V_{j} / c_{\infty}$ and static temperature ratio $T_{s, j} / T_{\infty}$ of the jet flow were monitored continuously, and data was only acquired while these two parameters were held within $0.5 \%$ of the set point in aggregate.

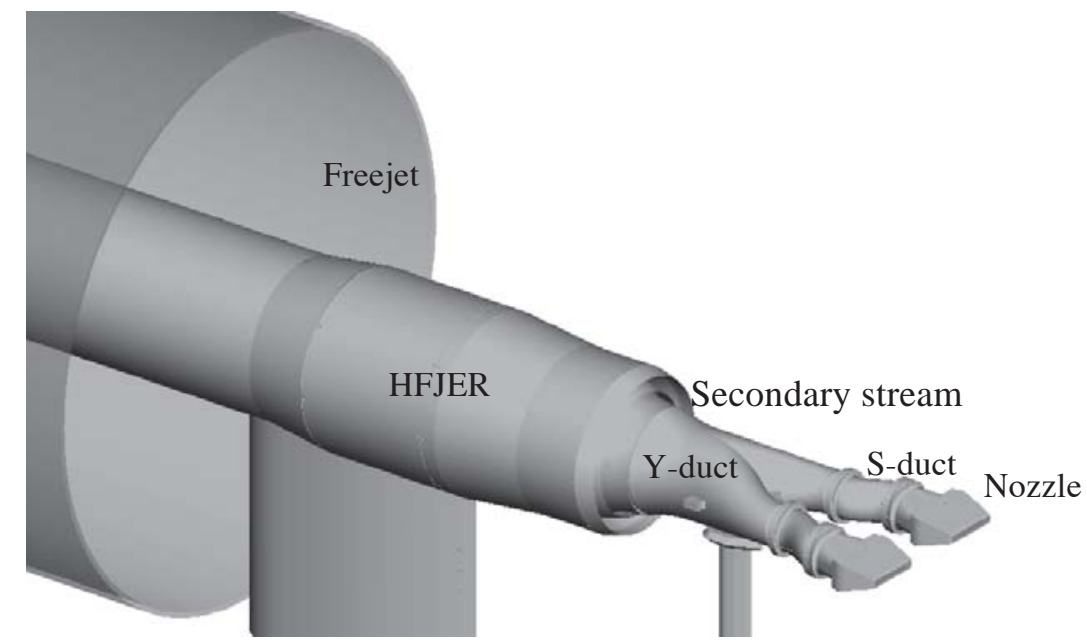

Figure 2 Twin model shown mounted on HFJER in the AAPL freejet.

Far-field acoustic data was acquired in both rigs on a radial arc of $1 / 4^{\prime \prime}$ Bruel \& Kjaer condenser microphones. The 24 microphones spanned an arc of $50^{\circ}-165^{\circ}$ in $5^{\circ}$ increments. In the SHJAR tests the microphone arc had a $150^{\prime \prime}(3.8 \mathrm{~m})$ radius; in the NATR tests the microphone arc had roughly a $46^{\prime}(14 \mathrm{~m})$ radius. These distances translate into radii of 75 and 275 jet diameters, respectively. Microphones were aimed at the nozzle exit and had grid caps removed. Microphones were individually calibrated for spectral response by the manufacturer within 6 months of the test and were calibrated using a pistonphone in situ just prior to the test.

All data presented in this paper have had the acoustic energy lost from atmospheric attenuation from the jet to the microphone added back to the spectrum, assuming spherical spreading from the nozzle exit 
to the microphone. All data have further been transformed to an arc with radius of 100 equivalent jet diameters under the same assumption.

\section{Model hardware}

\section{A. Nozzle hardware}

The model hardware used in these tests included five different pairs of nozzles along with the Y-duct. Two axisymmetric nozzles, SMC000 and TCON, were used, differing in their attachment flanges but otherwise similar in internal flow lines. All other nozzles were interchangeable between the two rigs.

The original rectangular nozzle family included nozzles of aspect ratio 2:1, 4:1, and 8:1. Details of their design are given in Ref 15 and initial acoustic results were documented in Ref 11. Key to the design of these nozzles was the lack of nonuniformity and swirl at the nozzle exit. Table 1 contains a list of the nozzles.

To achieve two identical streams to feed two nozzles at various spacings, the Y-duct and s-duct extensions were employed, as shown in Fig. 2. The s-ducts were rotated to change the nozzle separation distance. Note that there was two ways the s-ducts could be rotated to accomplish different separations. Previously ${ }^{14}$, the s-ducts were rotated in an anti-symmetric manner. Unfortunately, this method does not allow the rectangular nozzles to be aligned in a common plane parallel to the axes of the nozzles. Rotating the nozzles in opposite, symmetric directions does allow alignment of the rectangular nozzles and was used in this test after showing that the method of rotation produced little difference in the sound measurements.

Figure 3 illustrates the coordinate system used. To obtain a full azimuthal $(\phi)$ directivity mapping of the effect of one jet upon another, the Y-duct was clocked in $30^{\circ}$ increments relative to the microphone array. Similarly, when the rectangular nozzles were installed a similar clocking nomenclature was employed to measure azimuthal directivity of the jet sound field.

Table 1. Twin Model Nozzle Hardware

\begin{tabular}{|l|l|c|c|}
\hline \hline \multicolumn{1}{|c|}{ Description } & \multicolumn{1}{|c|}{ Name } & \multicolumn{1}{c|}{$\begin{array}{c}\mathbf{D}_{\mathbf{j}} \\
\text { inch }\end{array}$} & $\begin{array}{c}\text { Throat Area } \\
\text { inch }^{2}\end{array}$ \\
\hline \hline Conic Convergent & SMC000 & 2.000 & 3.142 \\
\hline Conic Convergent & TCON & 2.000 & 3.142 \\
\hline 2:1 Rectangle, Base & A2Z0 & 2.133 & 3.572 \\
\hline 8:1 Rectangle, Base & A8Z0 & 2.133 & 3.572 \\
\hline
\end{tabular}

Table 2. Nozzle Spacings

\begin{tabular}{|c|c|c|}
\hline \hline $\begin{array}{c}\text { Nozzle Spacing } \\
\text { inch }\end{array}$ & $\begin{array}{c}\text { S/D } \\
\text { Rectangle }\end{array}$ & $\begin{array}{c}\text { s/D } \\
\text { Round }\end{array}$ \\
\hline \hline 5.25 & 2.45 & 2.62 \\
\hline 8.25 & 3.85 & 4.12 \\
\hline 11.25 & 5.26 & 5.62 \\
\hline
\end{tabular}

\section{B. Surface hardware}

In addition to the nozzle hardware, the tests required a surface placed near the jet. The moveable surface used in previous SHJAR jet-surface interaction tests was the basis for the surface used here. To accommodate the differences in the rigs some differences were made between the rigs, but the changes were primarily in the method of mounting and actuating the surface. For instance, in the SHJAR test the 
surface was vertical with a significant carriage below to move the large surface. In the NATR test the surface was held at an angle of roughly $25^{\circ}$ to the horizontal to align with the overhead microphones. In both test rigs the surface was a $1 / 2$ " thick aluminum plate with a $45^{\circ}$ chisel bevel at the trailing edge. The surface plate was mounted to supporting structure at several points to minimize the chance for vibration of the plate. Aluminum tape was used to cover any screw holes or joints in the surface.

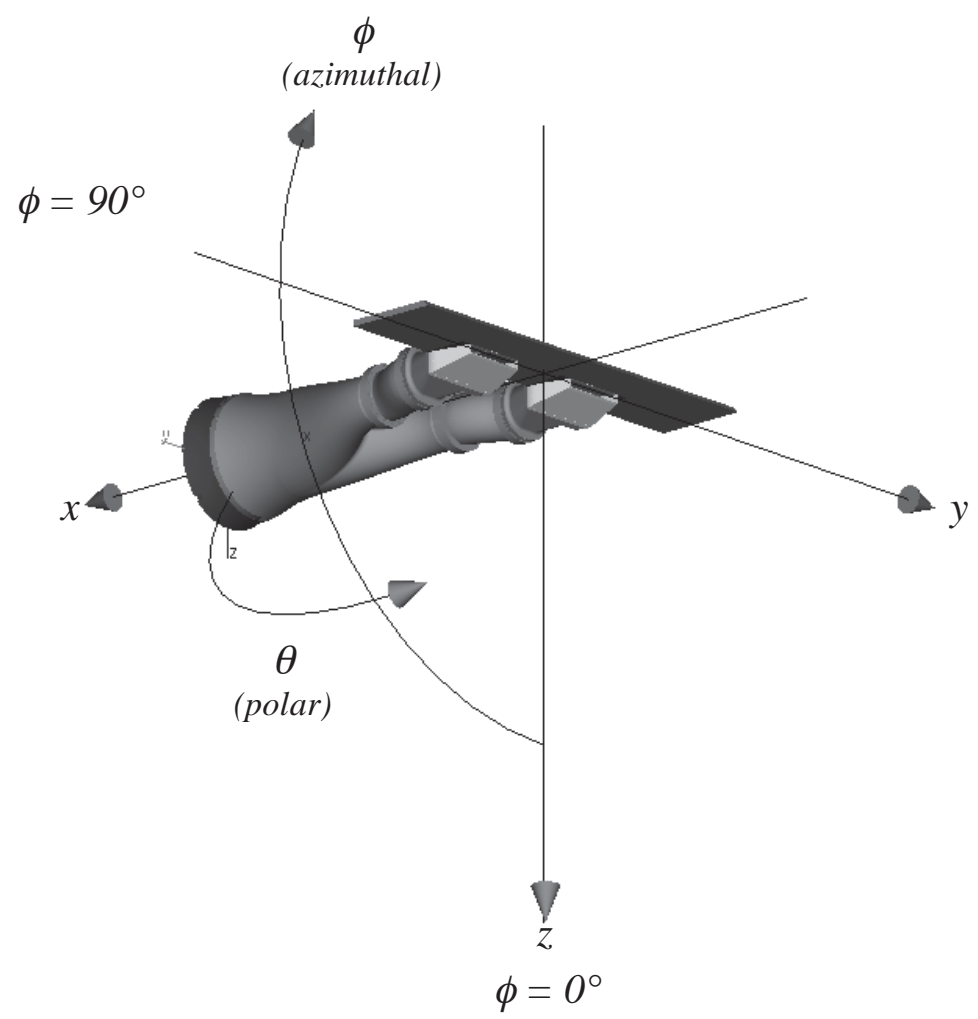

Figure 3 Model coordinates. Origin changes to nozzle centerline in case of single nozzle.

Figure 4 visually gives the definition of the surface position parameters, surface length and standoff. Note that the surface standoff $h$ is defined as the distance away from the lipline, $h=0$ being with the surface coplanar with the inside surface of the nozzle. Surface length $x_{T E}$ is measured from the nozzle exit plane to the downstream edge of the surface. Standoff distances and surface lengths to be used are given in Table 3 below. In the present tests, most configurations used the 24-inch span surface, with a few runs made with a 72-inch span surface to determine sensitivity to this feature. To allow the surface to reach the lipline the surface had to be cutback at the upstream edge for the nozzle body. This was done by replacing a section of the surface around the nozzle with a solid piece that complemented the nozzle outer surface. When the surface was at the nozzle lipline the nozzle and surface were essentially one piece. The entire surface was moved with a stepping motor control to achieve the various standoffs $h$ tested. The change in surface length $x_{T E}$ was accomplished by changing out the portion of the wall downstream of the nozzlematching piece with plates of different width (keeping the 24" span constant). The effect was to create multiple length surfaces which can be moved normal to the jet axis to achieve different standoff distances as shown in Figure 4. The final SHJAR setup, in both reflection and shielding orientations, is shown in Figure 5.

For NATR testing a few changes were made. First, the surface was used in the 'reflecting' configuration only. Second, the surface was extended to 36 inches in span to match the roughly 12 inches from the end of the surface to the centerline of the nozzles of the SHJAR configuration even when the nozzles are separated by 12 inches. Third, the range of widths and standoffs was reduced, as given in 
Table 3. One important note: only static (no freejet), cold flow conditions were tested with the surface in place. To produce some data for surfaces with flight effect the beveled rectangular nozzles were tested with forward flight; however, these only gave data for $h=0$ and $x_{T E}=2.7$ ".

Figure 6 shows the actuated surface as installed on NATR, emphasizing the orientation required for the $90^{\circ}$ clocking of the twin rig.

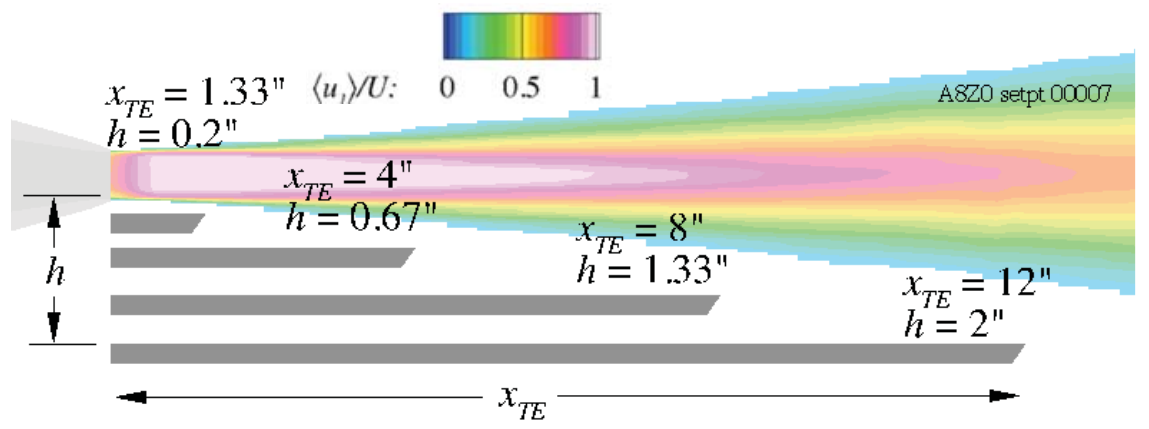

Figure 4 Definition of surface variables surface length $\left(x_{T E}\right)$ and standoff $(h)$.

Table 3 Surface positions used comprised of combinations of these parameters.

\begin{tabular}{|cc|c|cc|}
\hline \hline $\boldsymbol{x}_{\boldsymbol{T E}}$ (inch) & $\boldsymbol{x}_{\boldsymbol{T E}} / \boldsymbol{D}$ & & $\boldsymbol{h}$ (inch) & $\boldsymbol{h} / \boldsymbol{D}$ \\
\hline \hline 1.30 & 0.65 & & 0 & 0 \\
2.70 & 1.35 & & 0.2 & 0.1 \\
4.00 & 2 & & 0.5 & 0.25 \\
8.00 & 4 & & 1 & 0.5 \\
12.00 & 6 & & 1.9 & 0.95 \\
& & & 3.2 & 1.6 \\
\hline
\end{tabular}
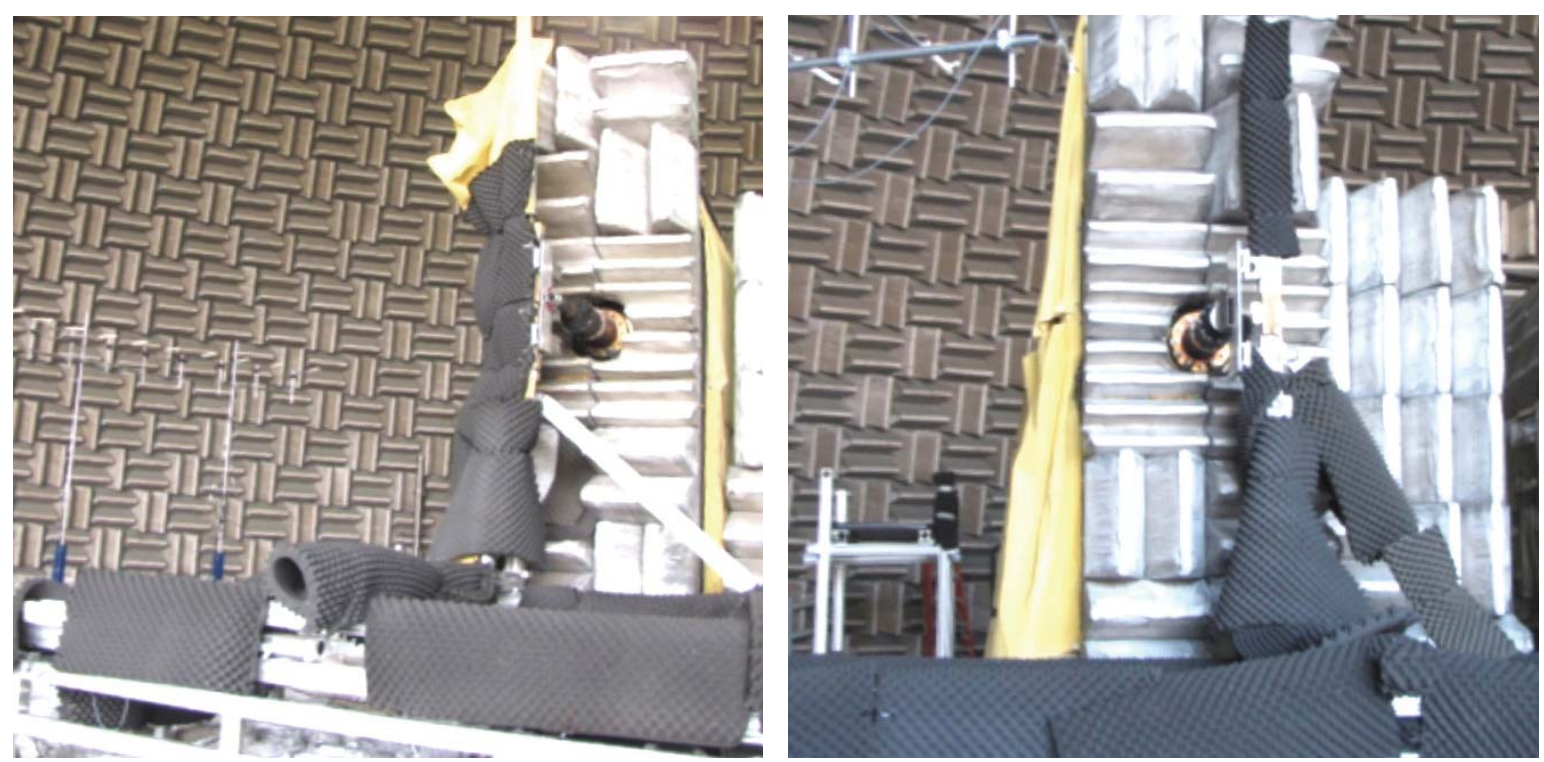

Figure 5 Jet-surface configurations on SHJAR: 'shielding' (left), reflecting (right). 2-foot span wall with low profile supports and pulled back foam to minimize blockage of upstream microphones. 


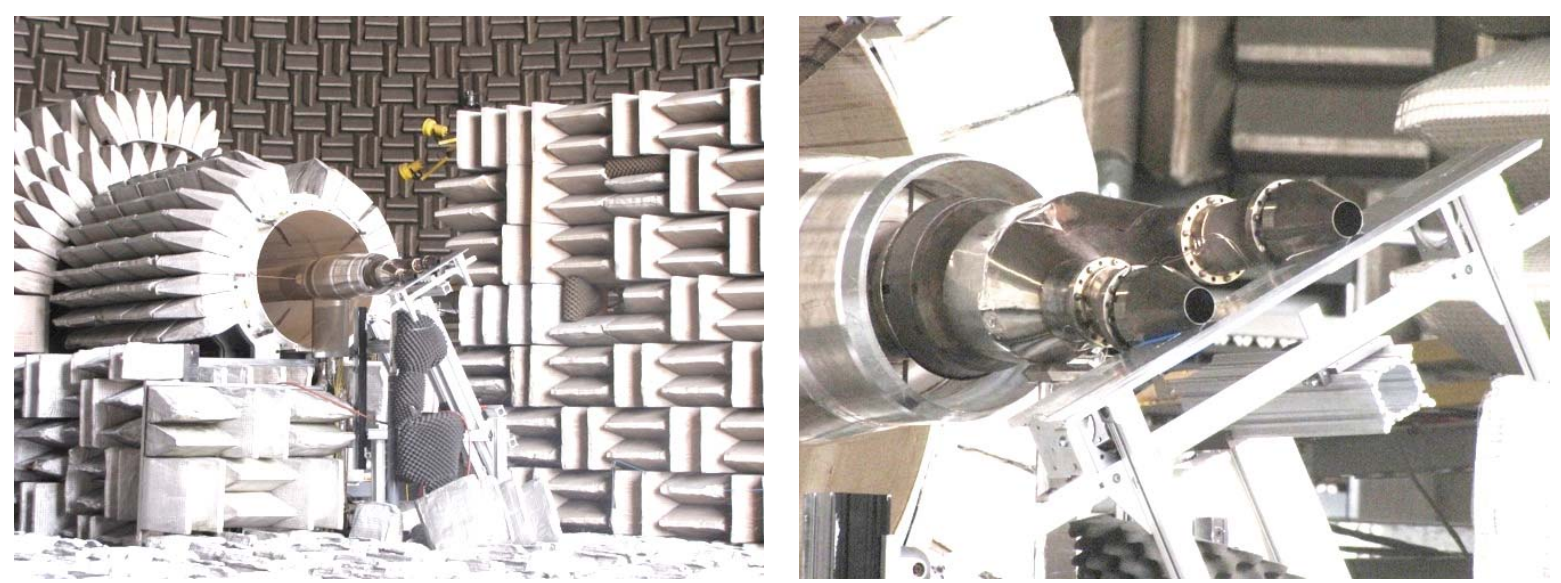

Figure 6 Jet-surface configuration on NATR. Surface and support holding surface at $30^{\circ}$ to horizontal (90 clocking) on twin nozzle config on NATR. Microphones are overhead in a plane normal to the plate.

Finally, to allow direct comparison of data acquired on NATR with the single jet-surface data acquired on SHJAR, configurations were run on NATR with a single nozzle, the second nozzle being replaced with a blank-off plate and cone, and the slot on the surface being filled with a simple piece of stock to be flush with the remaining plate. The blank-off plate and tapered cone shown in Figure 7 were also used to obtain data for rectangular nozzles in forward flight and to cross-check single-nozzle results between the two rigs.

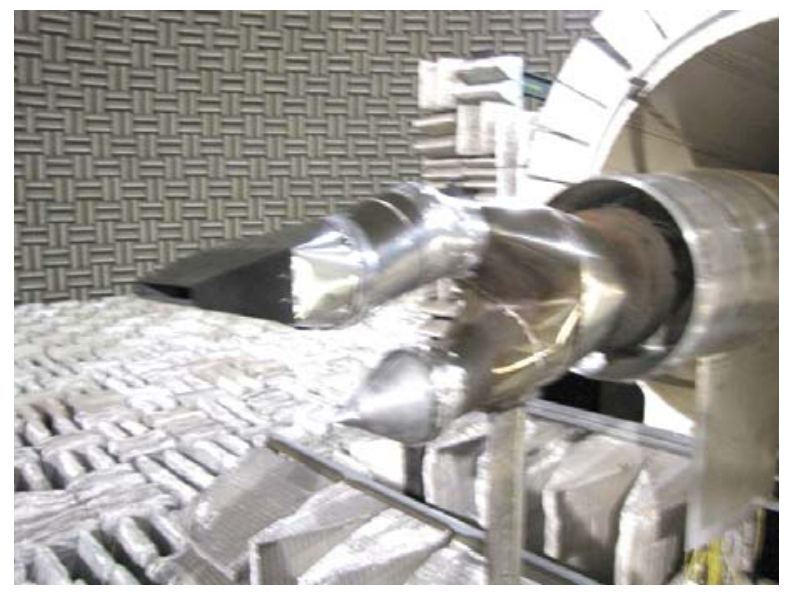

Figure 7 Blank-off plate and tapered closeout on lower leg of y-duct, used to obtain single-nozzle data (rectangular nozzle in this picture) on HFJER.

\section{Flow Conditions}

Because of the large number of geometric variations studied only a limited number of flow conditions were used. It has been shown ${ }^{16}$ as far back as the 1970's that by fixing acoustic Mach number Ma and static temperature ratio $T_{s, j} j T_{\infty}$ jet noise is very repeatable, independent of ambient conditions once atmospheric attenuation over the propagating distance from the jet to the microphone has been accounted for. Thus the matrix of setpoints listed in Table 4 was employed. There were some exceptions to this: configurations involving surfaces were not tested with hot flows, nor were they tested in with simulated flight. The surface hardware featured an ABS plastic piece fitted to the outside of the nozzle, and the mechanism for moving the surface was not strong enough to withstand the aero load of flight. There was also a minimum jet Mach number could be measured for a given simulated flight speed. Noise of the freejet providing the simulated flight is louder than the model nozzle jet for $M a<0.9$, limiting the low speeds measured. 
Table 4 Flow conditions of the SHJAR and HFJER tests

\begin{tabular}{ccrrrc}
\hline \hline Setpoint & $N P R$ & $N T R$ & $M_{f i}$ & $M a$ & $T_{s, j} / T_{\infty}$ \\
\hline \hline 30 & 1.197 & 1 & 0 & 0.500 & 0.95 \\
50 & 1.435 & 1 & 0 & 0.700 & 0.90 \\
70 & 1.855 & 1 & 0 & 0.900 & 0.84 \\
270 & 1.361 & 1.920 & 0 & 0.900 & 1.76 \\
271 & 1.361 & 1.920 & 0.1 & 0.900 & 1.76 \\
272 & 1.361 & 1.920 & 0.2 & 0.900 & 1.76 \\
273 & 1.361 & 1.920 & 0.25 & 0.900 & 1.76 \\
290 & 1.896 & 2.110 & 0 & 1.329 & 1.76 \\
291 & 1.896 & 2.110 & 0.1 & 1.329 & 1.76 \\
292 & 1.896 & 2.110 & 0.2 & 1.329 & 1.76 \\
293 & 1.896 & 2.110 & 0.25 & 1.329 & 1.76 \\
360 & 1.274 & 2.422 & 0 & 0.900 & 2.27 \\
380 & 1.663 & 2.608 & 0 & 1.330 & 2.27
\end{tabular}

\section{Sample Experimental Results}

In the following figures the acoustic impact of variations in the test configurations are presented as carpet plots of power spectral density as a function of frequency in Strouhal number $\left(f D_{e} / U\right)$ and polar angle $\theta$ measured from the forward flight axis. The data have been transformed to a common observer distance of 100 nozzle diameters with atmospheric attenuation added back to the spectra. Most of the data have been averaged into $1 / 12^{\text {th }}$ octave bandwidths to reduce the random error that is magnified when two narrowband spectra are subtracted.

Data presentation will follow the demonstration of meeting test objectives, with the first few sections devoted to affirming that the data was consistent between rigs and between test entries. Later sections give a taste of the findings regarding the impact of various geometric parameters documented in the test.

\section{A. Objective 1: Document differences in rigs with single jet}

The two rigs used in these tests are located in the same facility and share data acquisition systems. However, their anechoic fields, air supply systems, microphone arrays, and round nozzle contours are significantly different. To be sure that results obtained in one rig were pertinent to the other, several comparisons were made between the two rigs. The most obvious method is to subtract the spectral directivity (in 1/12 octave bandwidths) for data acquired at the same flow conditions. The difference, in $\triangle \mathrm{dB}$, HFJER - SHJAR, is plotted as color on a carpet plot of the spectral directivity of the SHJAR data in Figure 8.

From the figure the difference is generally less than $\pm 1 \mathrm{~dB}$ and less than $\pm 0.5 \mathrm{~dB}$ in the frequencies and angles of prime interest. From this it was concluded that the two rigs gave comparable results within $1 \mathrm{~dB}$ for a single nozzle flow. To eliminate even this bias, studies involving effects of second jets and forward flight should use a baseline dataset from the same rig. 
(a)

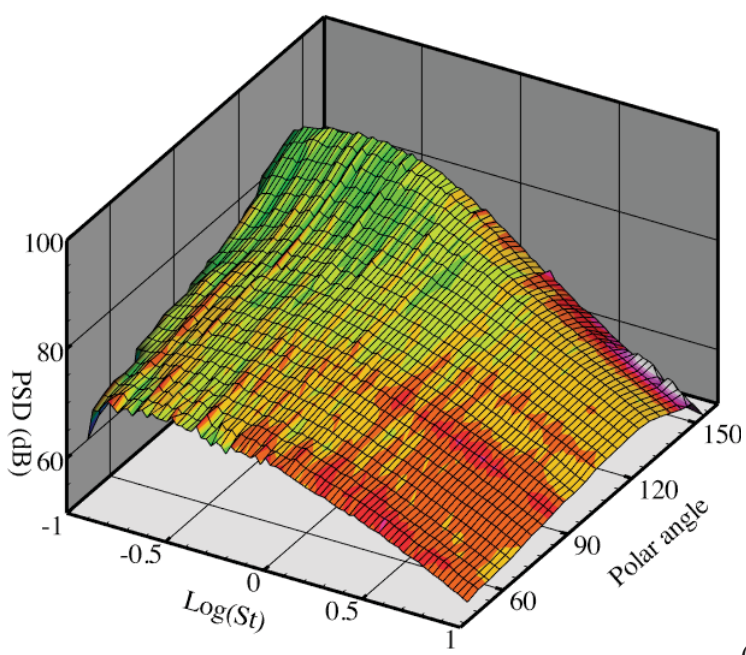

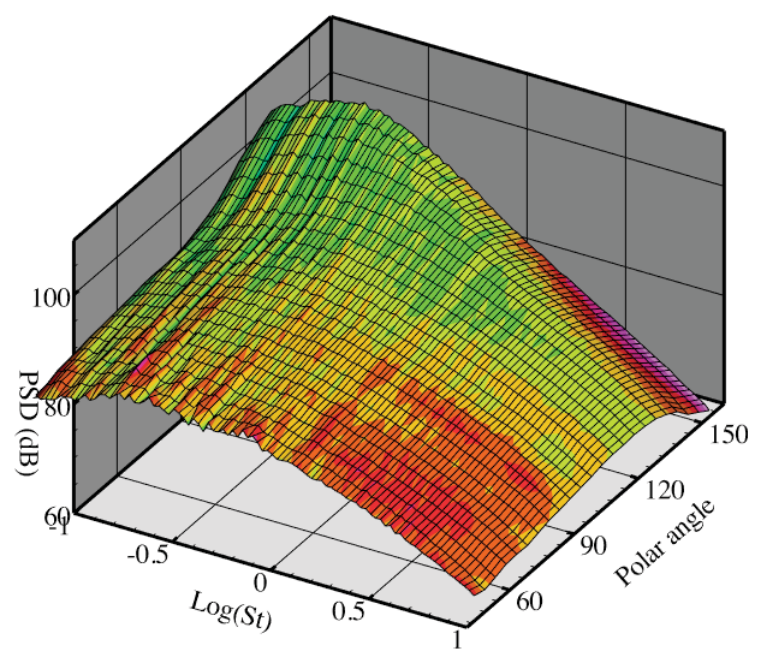

(b)

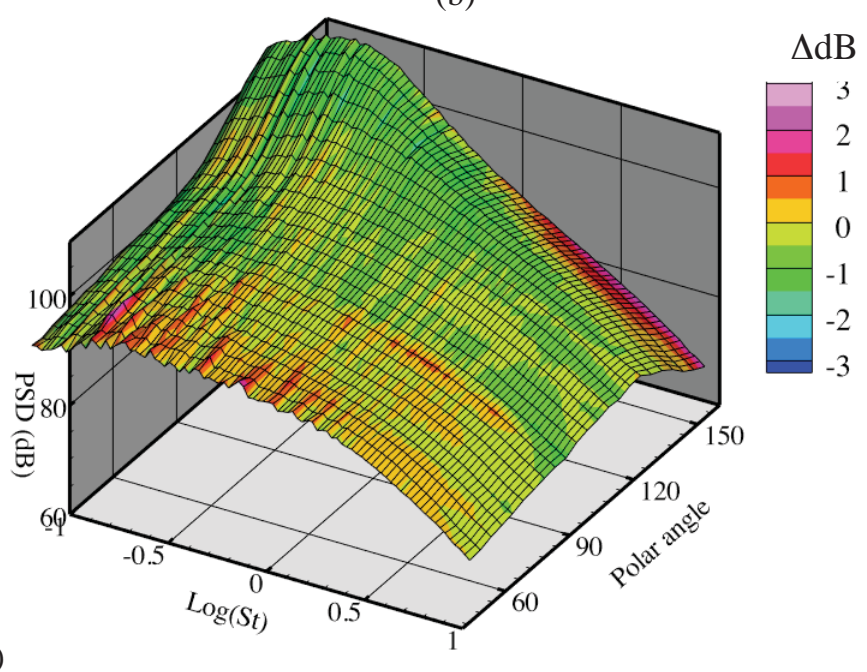

(c)

Figure 8 Difference in spectral directivity of round nozzles measured on SHJAR and on HFJER at three unheated conditions: (a) $M a=0.5$, (b) $M a=0.7$, (c) $M a=0.9$.

\section{B. Objective 2: Document the sensitivity to surface span on the jet-surface interaction effect}

In the tests the span of the surface is supposed to be large enough that its dimension didn't matter ('semi-infinite'). To determine to what extent this was the case for the 24" span used, a second surface measuring 72" in span was used for a limited number of configurations. All other aspects of the plate were the same including the $x_{T E}=12$ " length and the edge bevel. The acoustic spectral directivities of a jet interacting with the two different spans were compared by direct subtraction and are presented in Figure 9.

The difference in noise produced by the two different spans was generally less than $0.5 \mathrm{~dB}$, with most differences being in the spectral character associated with the trailing edge dipole. Consistent differences were seen first when the surface had zero standoff from the jet lipline, where the larger span produced $2 \mathrm{~dB}$ more noise than the smaller one. This was not found for other standoff heights. The second consistent difference was found at forward angles and high frequencies where the smaller span produced $1 \mathrm{~dB}-1.5 \mathrm{~dB}$ more sound than the larger span configuration. The final consistent difference, and possibly most important, is a series of fat tones that were produced with the larger span surface. These spectral details are most apparent at angles broadside to the jet and occur near the peak frequency of the jet 
spectra. These tones were not the result of an aeroelastic response of the larger span surface as determined by separate exploratory tests ${ }^{17}$.

(a)
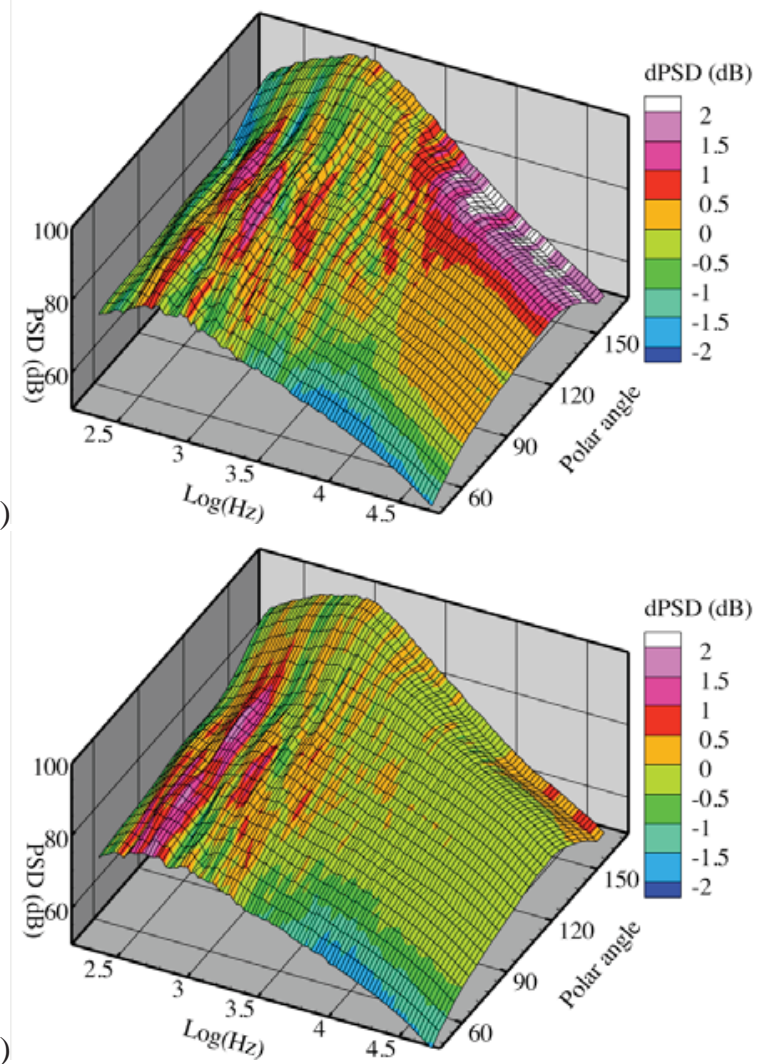

(b)

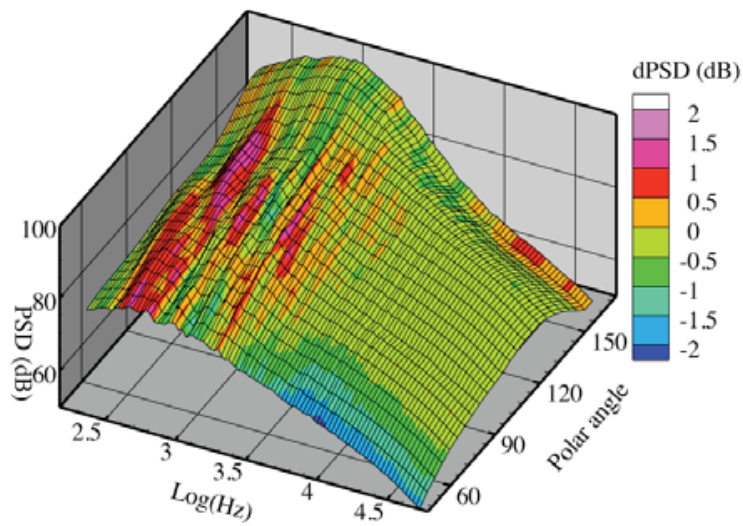

(d)

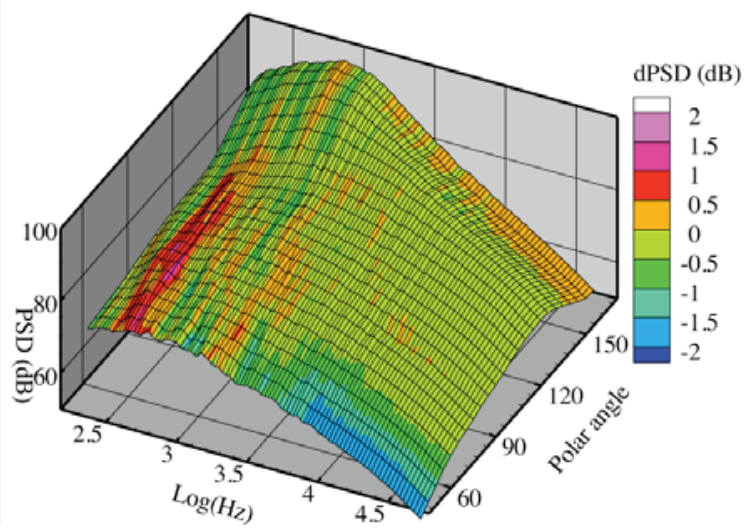

(e)

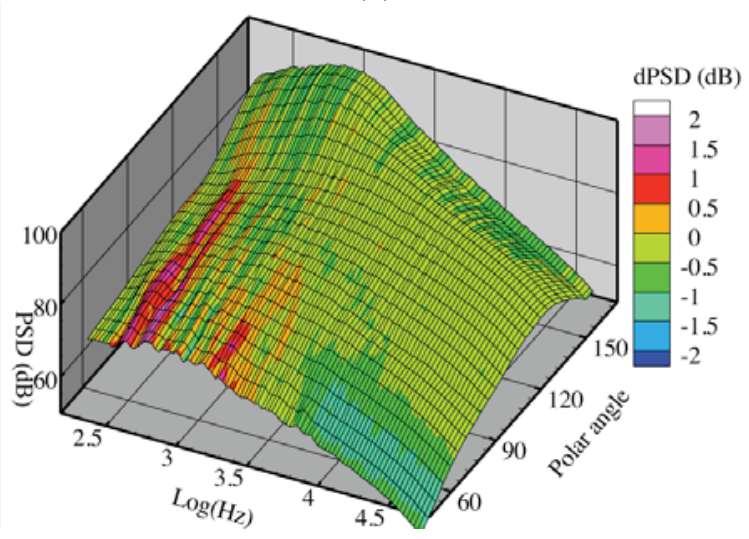

Figure 9 Effect of surface span (distance to edge normal to jet axis, 2'vs 6' surface) on spectral directivity of jet-surface interaction noise. Colors are difference in power spectral density $(d B), 24$ " 72”. Nozzle SMCOOO unheated at Ma $=0.9$ with $x_{T E}=12$ " and different standoffs from lip: $h / D=(a)$ 0.0, (b) 1.0, (c) 2.0, (d) 4.0, (e) 10.0.

\section{Objective 3: Confirm that the acoustic impact of a surface near a single jet is the same in both rigs.}

In the SHJAR tests a substantial effort went into creating a semi-infinite surface for the jet-surface interaction measurements. In the HFJER test the surface was much less extensive and data was only collected in a $\phi=0^{\circ}$ plane (the 'reflected' side of the surface). The acoustic impact of the surface was extracted by directly subtracting the power spectral density of the isolated jet from that of the jet with 
surface in $1 / 12^{\text {th }}$ octave bandwidths. For greater data density on the page, the spectral directivity of the difference, with surface minus without, will be given as a two-dimensional contour plot with polar angle on the vertical axis and the logarithm of Strouhal number on the horizontal axis, as shown in Figure 10.

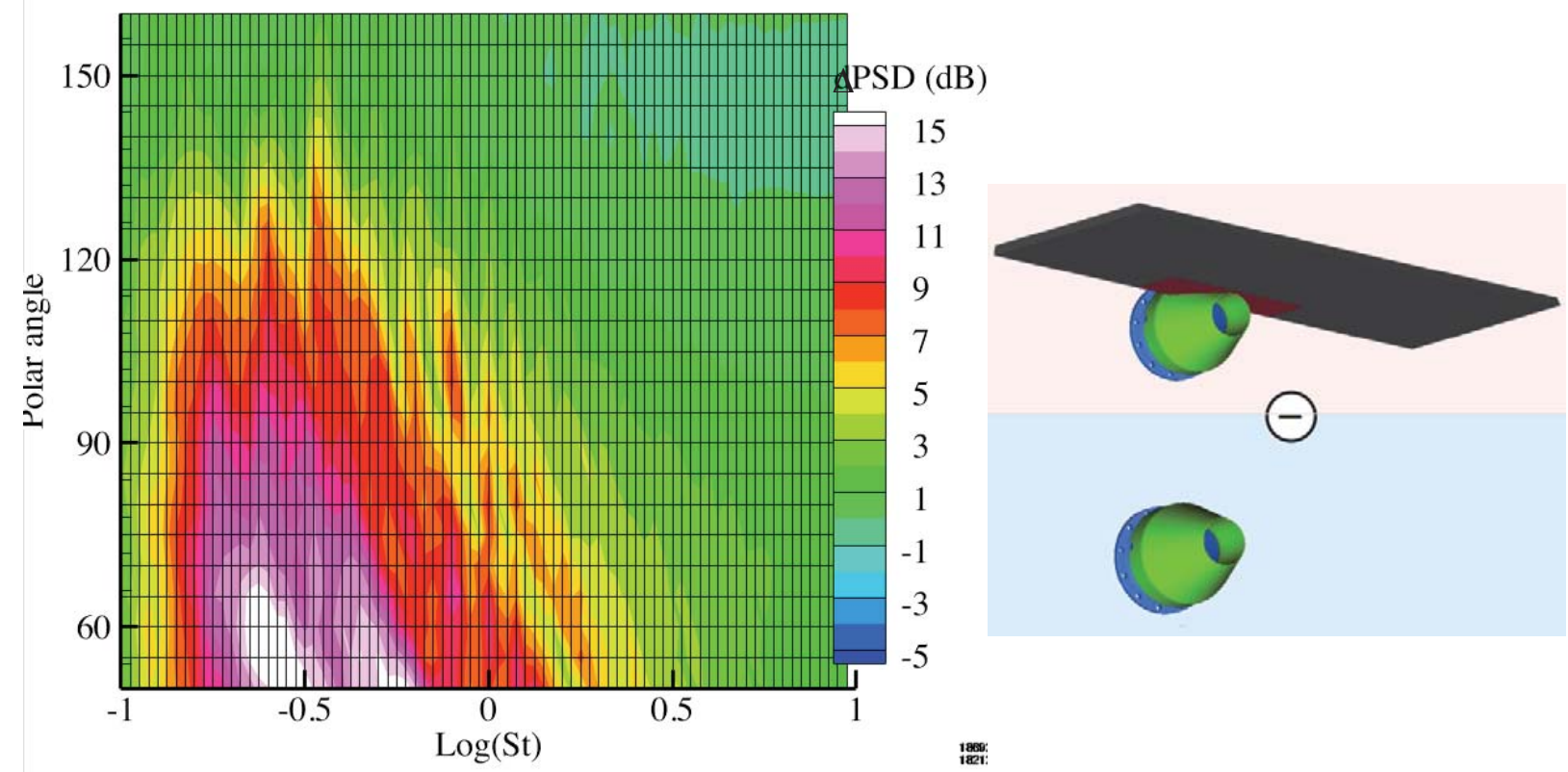

Figure 10 Typical contour plot of difference in sound, with surface minus without surface. This plot is for unheated round jet at Ma $=0.5$ with and without surface at standoff $h=0$ and length $x_{T E}=4$ ". Plot format is used in subsequent composite maps where surface parameters are varied.

To see how the noise difference caused by changes in the surface parameters of standoff and length the contour plots are arranged in rows of a constant surface length $x_{T E}$ and columns of constant standoff $h$ as shown in Figure 11 and Figure 12. The former present results from the SHJAR test and the latter from the HFJER test. As a reminder, these tests differed in the axisymmetric nozzle used (SMC000 vs TCON), the details of the surface, and the measurement distance. From the plots below the difference produced by the surface measured in the two tests agreed to within $1 \mathrm{~dB}$. 

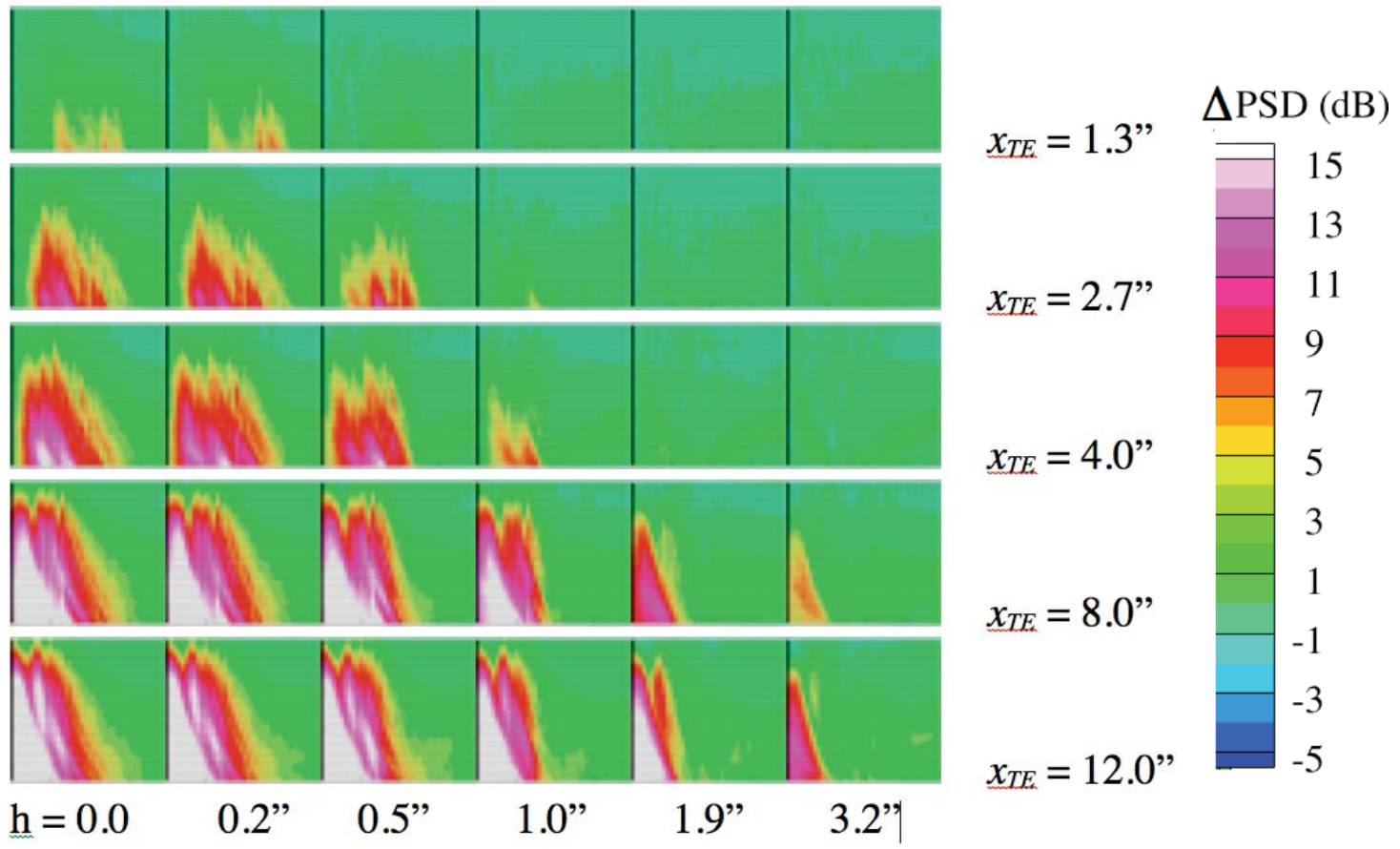

$\mathrm{h}=0.0 \quad 0.2 " \quad 0.5 " \quad 1.0 " \quad 1.9 " \quad 3.2$ "

Figure 11 Aggregate of maps showing impact of jet-surface interaction on sound. Each cell is a map of difference in noise, with surface minus without, as shown in detail in Figure 10, for an observer on the reflecting side of the jet $\left(\phi=0^{\circ}\right)$. Data from round SMCOOO nozzle in SHJAR test.
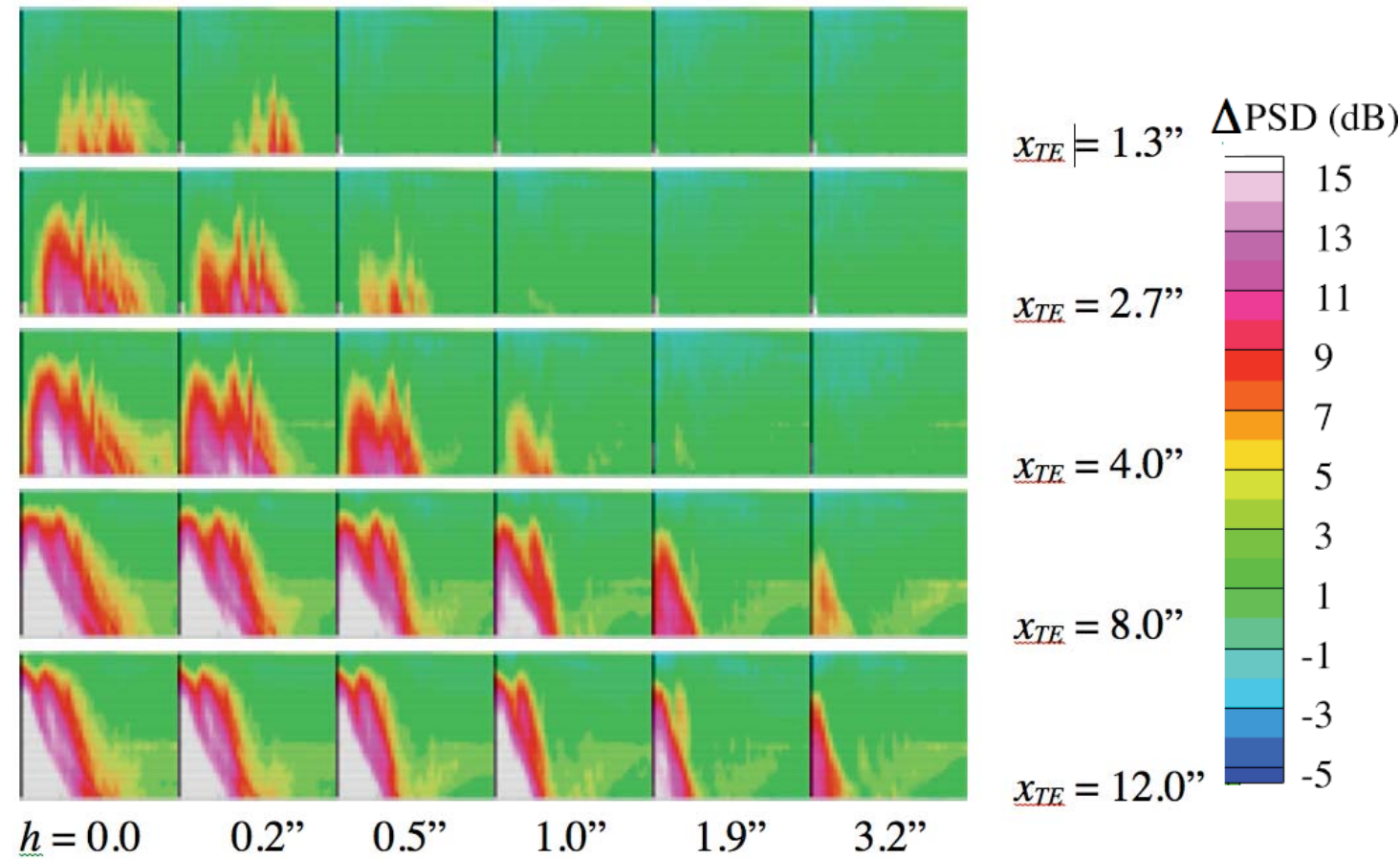

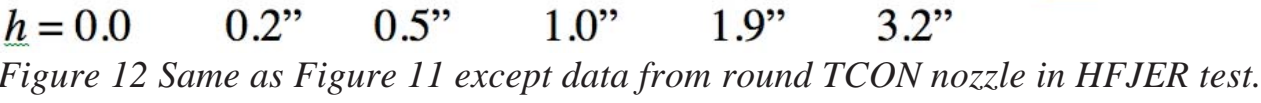

D. Objective 4: Determine the impact of jet aspect ratio on the acoustic impact of a surface near a single jet.

Similar to the data just presented for the round jet, the impact of a nearby surface on a 8:1 rectangular jet is given in Figure 13. The difference in noise, with surface minus without, can be compared with the 
figures directly above for the round jet. Although there were differences they were small, with the rectangular nozzle having slightly larger differences, both positive and negative. For instance, there is more blue at aft angles and low frequencies in the plots of short surfaces, indicating that the surface reduced noise there. This was atop the reduction at these angles and frequencies brought about by increasing the aspect ratio of the nozzle.

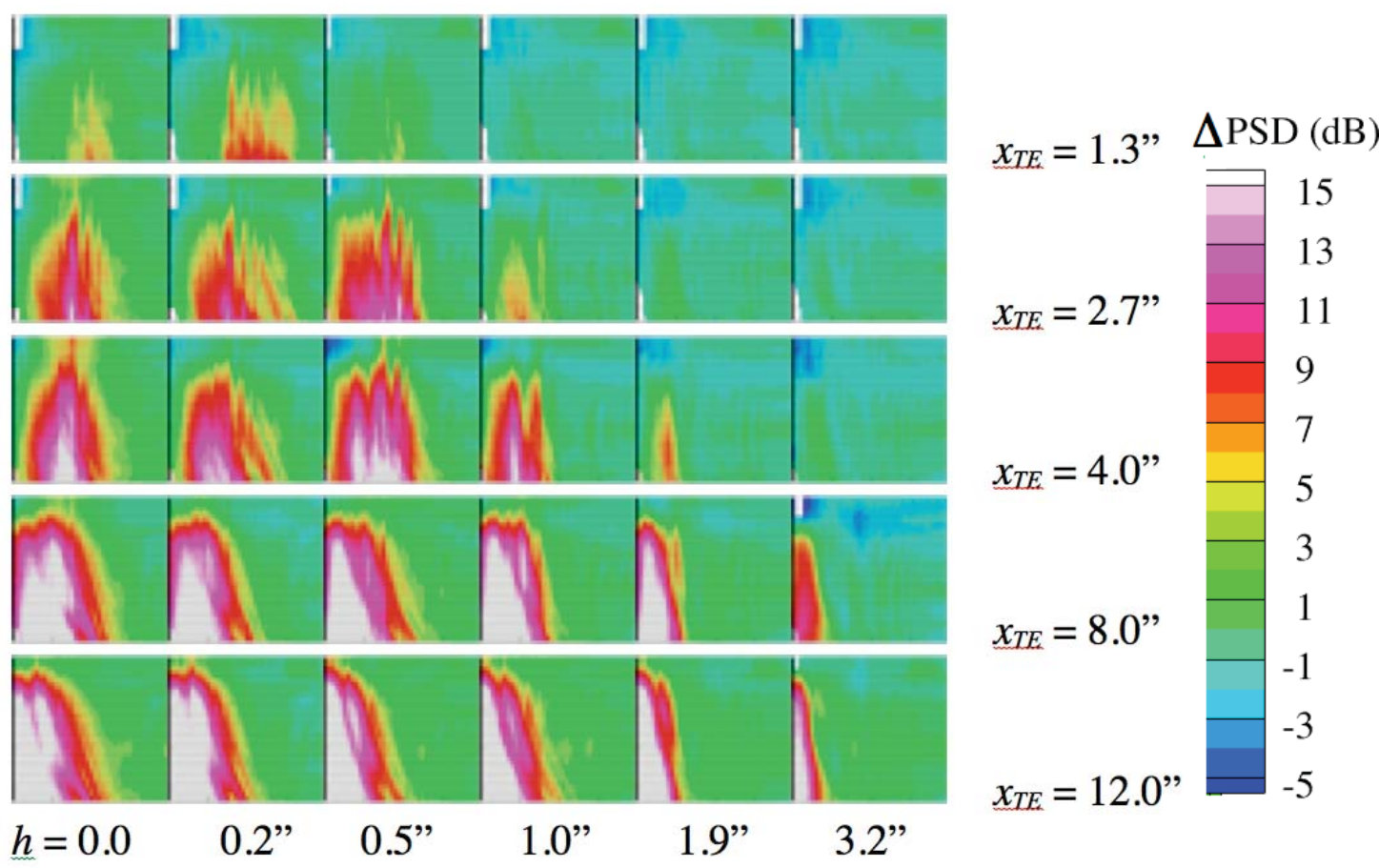

Figure 13 Same as Figure 12 except data from rectangular A8Z0 nozzle.

\section{E. Objective 5: Determine the impact of jet aspect ratio on the forward flight effect.}

It is well known that radiated noise from round jets decreases with increasing flight speed. High aspect ratio nozzles have more wetted area and may react to the ambient velocity differently than a round nozzle of similar throat area. Figure 14 gives a direct measure of the flight effect on a round jet by subtracting the noise of the static round jet from the jet in a $M_{\text {flight }}=0.25$ flight stream. Figure 15 and Figure 16 show the same analysis for the $2: 1$ and $8: 1$ rectangular jets.

At broadside angles the impact of forward flight was very similar on all the jets, with reductions of $3 \mathrm{~dB}$ at peak frequencies and less at higher frequencies. Round jets in a flight stream of $M_{\text {flight }}=0.25$ produced $6 \mathrm{~dB}$ less noise than static jets around the peak downstream angle and peak frequency. This difference decreased with increasing aspect ratio to become only 3-4dB in the 8:1 jet. Although the 2:1 jet had a nearly axisymmetric flight effect, the effect was strongly asymmetric in the aft angles of the 8:1 jet. High frequencies at aft angles, which can be elevated in rectangular jets compared to a round jet, are suppressed by $6 \mathrm{~dB}$ on the wide side of the nozzle, but only $4 \mathrm{~dB}$ on the narrow side. 


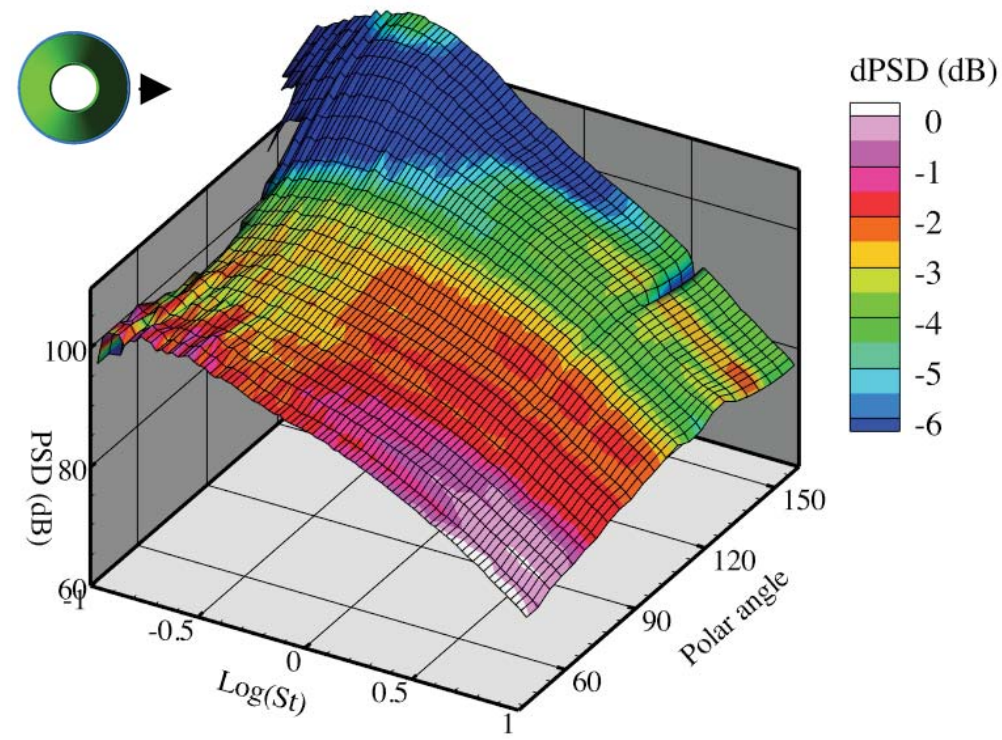

Figure 14 The effect of flight on spectral directivity of noise from a single round nozzle (TCON). Jet flow is $M a=1.33, \mathrm{~T}_{\mathrm{s},} / \mathrm{T}_{\infty}=1.76$. Sound from jet in flight $M_{\text {flight }}=0.25$ minus sound with no forward flight .
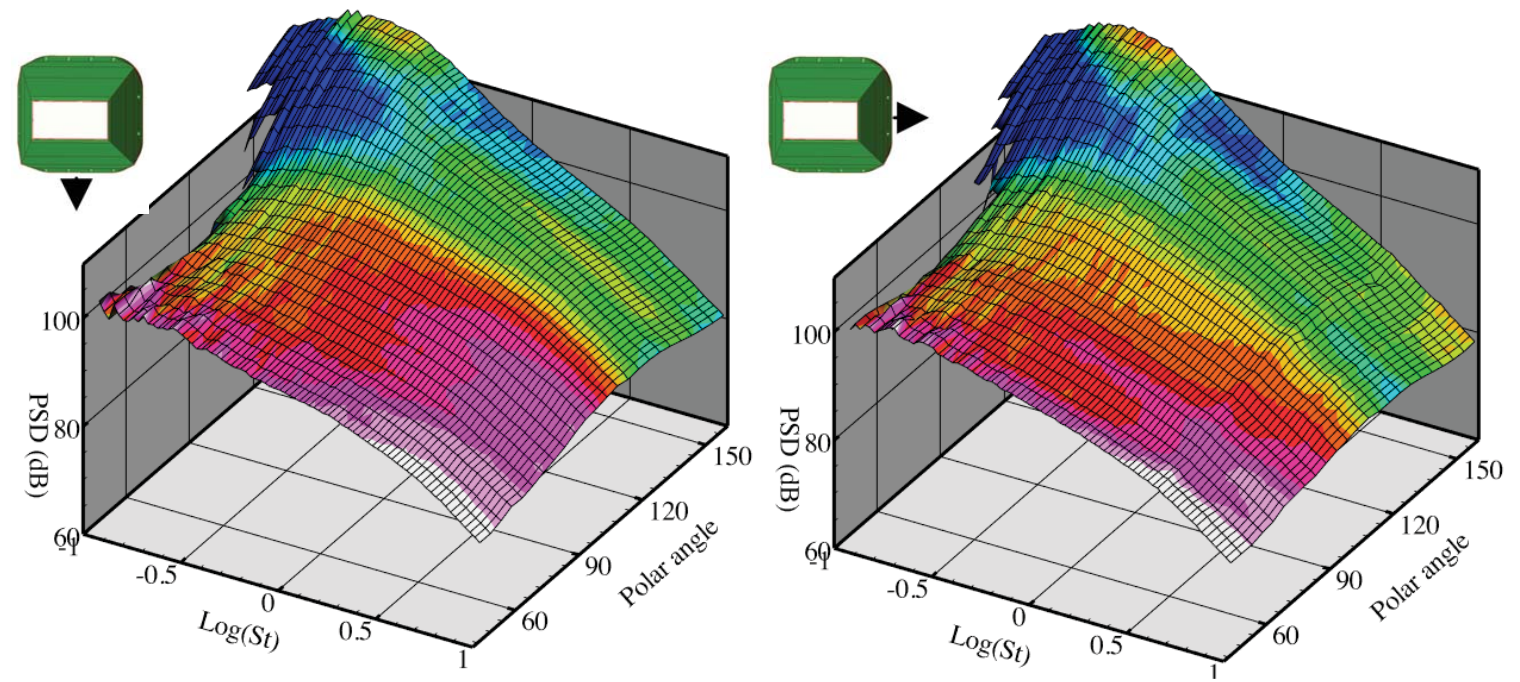

Figure 15 Same as Figure 14 except nozzle is A2Z0 with observers in $\phi=0^{\circ}$ plane (left) and $\phi=90^{\circ}$ plane (right). 

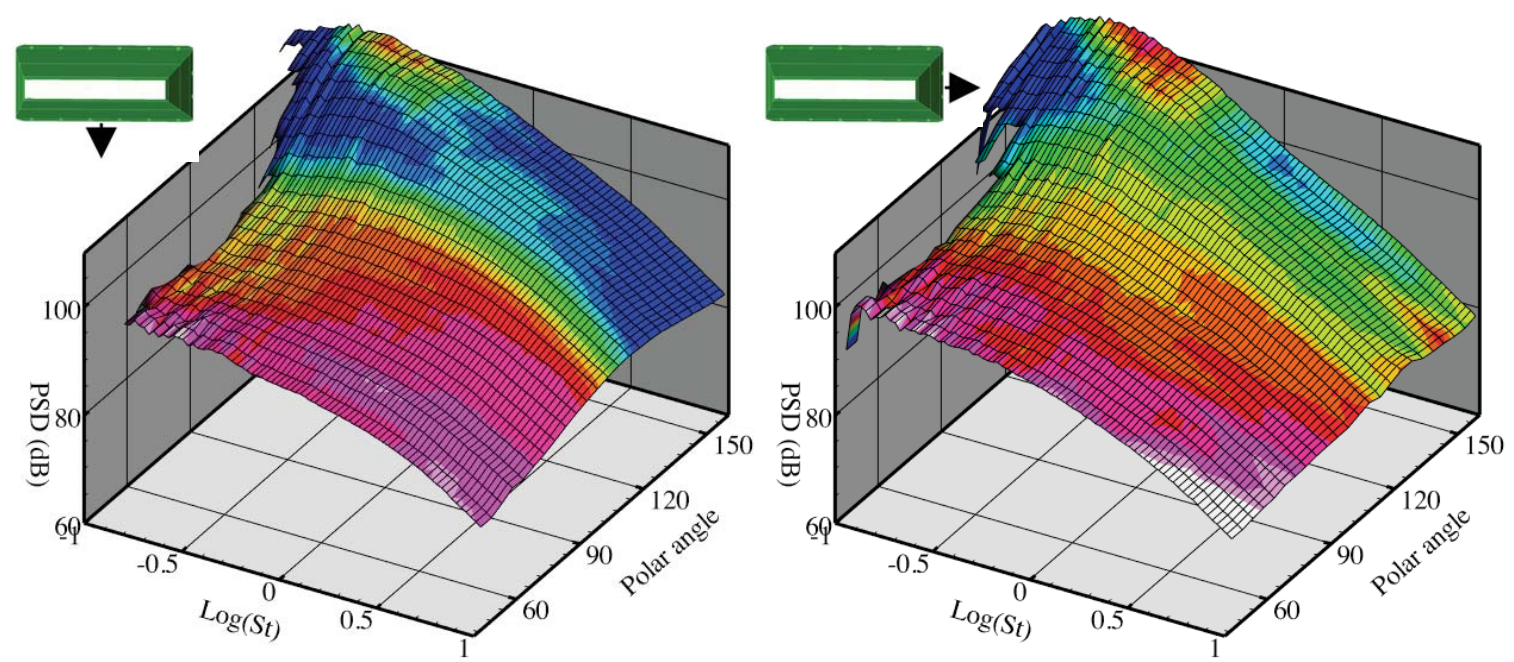

Figure 16 Same as Figure 14 except nozzle is A8Z0 with observers in $\phi=0^{\circ}$ plane (left) and $\phi=90^{\circ}$ plane (right).

\section{F. Objective 6: Confirm impact of jet spacing on the acoustic spectral directivity from twin round jets.}

In a previous test at NATR using the same Y-duct, but different ambient flow hardware, the effect of adding a second jet was documented ${ }^{14}$. There were some interesting observations made from that test that needed to be confirmed in a second test with cleaner ambient flow. From basic acoustic understanding the main impact of adding a second incoherent jet noise source should be to add $3 \mathrm{~dB}$ to the measured total sound. The testing found a much more complicated situation.

Figure 17 presents data from the previous (TWN10) test at flow conditions very near to those acquired in the present test. The shapes of the carpet plots are the acoustic spectral directivity produced by two jets for the closest spacing tested, $s / D=2.62$. The color contours represent the difference in spectral directivity between dual and single jets. At an azimuthal angle $\phi=0^{\circ}$ there was an interesting dual peak in the spectral directivity of the two jets, the addition of the second jet adding $6 \mathrm{~dB}$ to the sound of one jet alone. At $\phi=90^{\circ}$ the broadside angles showed an increase of roughly $3 \mathrm{~dB}$, but at aft angles adding a second jet only increased the sound by $0-2 \mathrm{~dB}$.
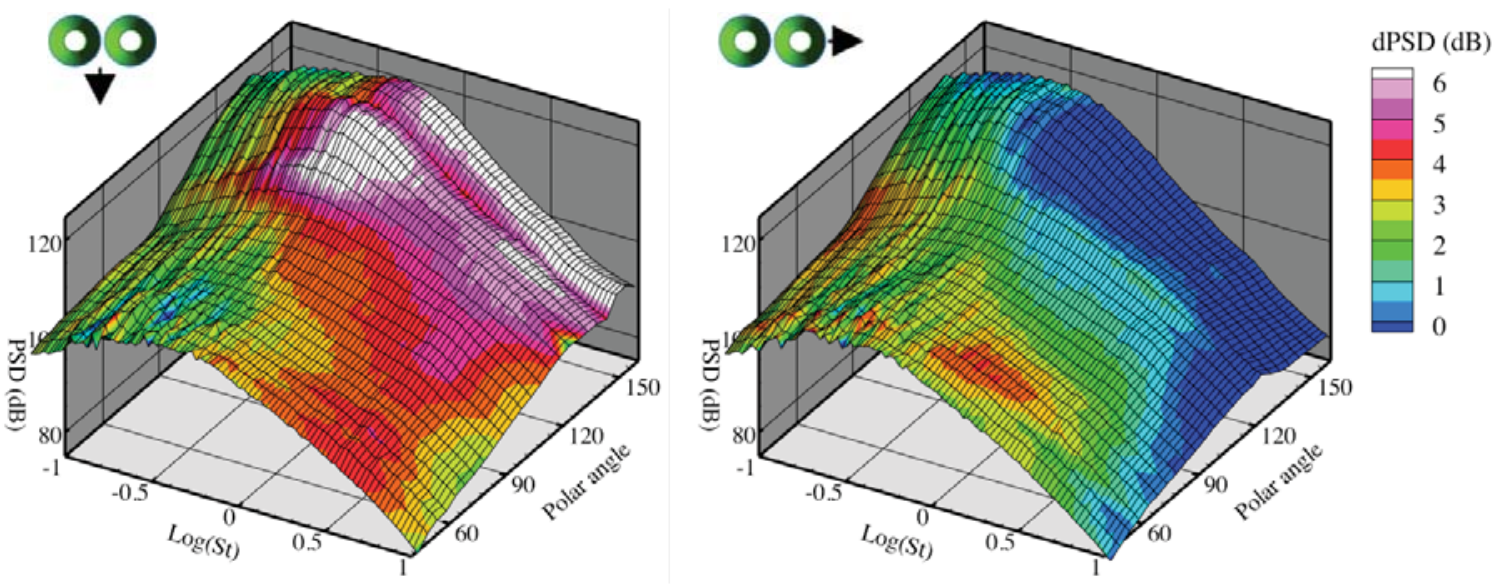

Figure 17 Difference in acoustic spectral directivity caused by adding a second round jet with spacing $s / D=2.62$ as seen by observer normal to their common plane $\left(\phi=0^{\circ}\right.$; left $)$ and in line with jets $\left(\phi=90^{\circ}\right.$; right). Shape is spectral directivity of two jets. Flow condition is $M a=1.40, \mathrm{~T}_{\mathrm{s}, \mathrm{j}} / \mathrm{T}_{\infty}=2.00, M_{\text {flight }}=0.1$. 
The present test data, Figure 18, shows the same trends, with a second peak in the $\phi=0^{\circ}$ plane and very little additional noise in the $\phi=90^{\circ}$ plane. Comparing the two azimuthal angles, the impact of adding a second jet produced a $6 \mathrm{~dB}$ asymmetry at the downstream, peak noise polar angles.
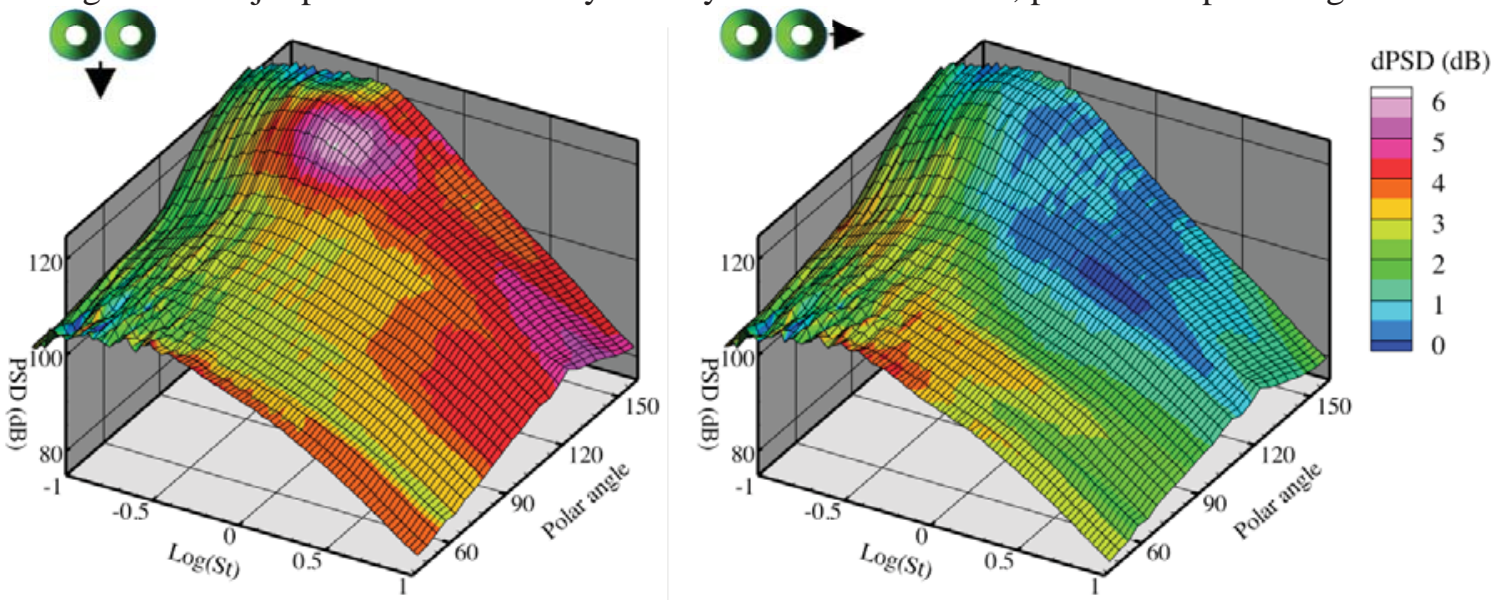

Figure 18 Same as Figure 17 except data from present tests and flow condition is $M a=1.33, \mathrm{~T}_{\mathrm{s}, \mathrm{j}} / \mathrm{T}_{\infty}=$ 1.76, $M_{\text {flight }}=0.1$.

The findings for the widest spacing measured, $s / D=5.62$, were similarly in agreement between the two tests. As seen in Figure 19 (TWN10 test) and Figure 20 (current test), the inclusion of a second jet created a 'valley' at roughly $\mathrm{St}=1$ across the peak polar angles at $\phi=0^{\circ}$, and increased noise at the peak by $6 \mathrm{~dB}$ over the single jet. At $\phi=90^{\circ}$ the impact of the second jet was nearly the same as at the closer spacing, generally adding little to no additional noise at aft angles.
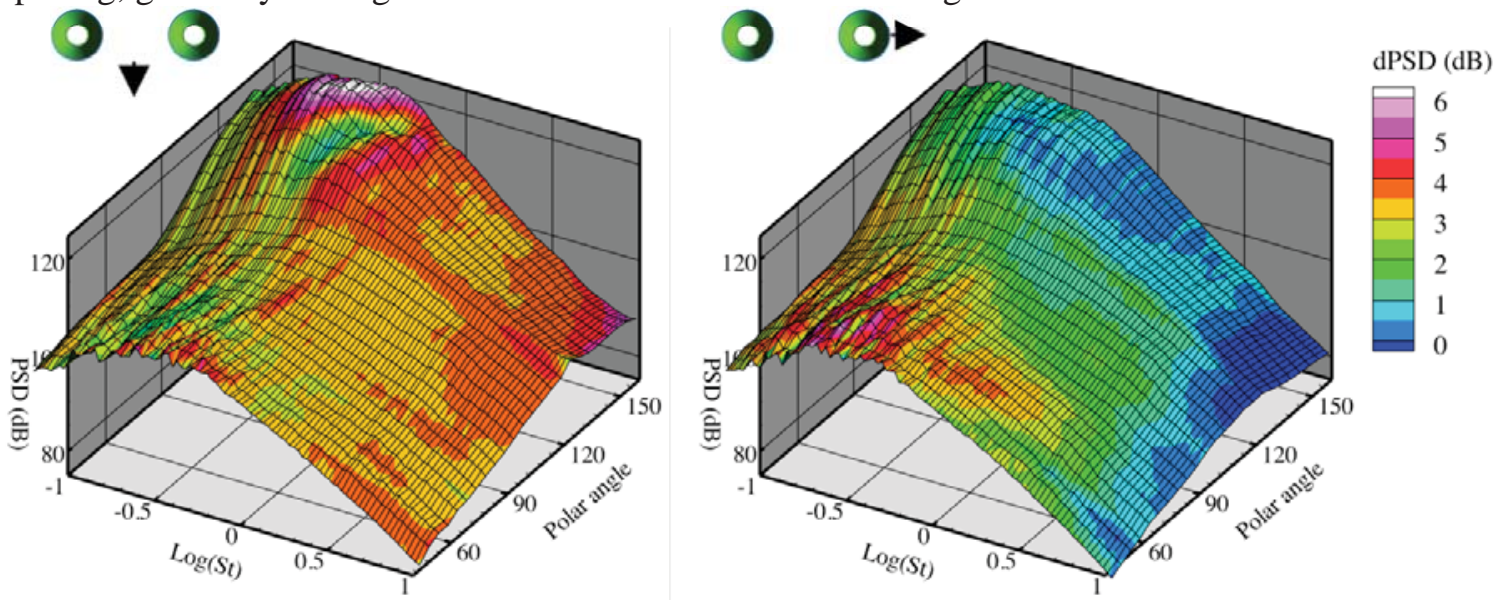

Figure 19 Difference in spectral directivity caused by second round jet with spacing $s / D=5.62$ as seen by observer normal to their common plane $\left(\phi=0^{\circ}\right.$; left $)$ and in line with jets $\left(\phi=90^{\circ}\right.$; right $)$. Data acquired in TWN10 test. Shape is spectral directivity of two jets. Flow condition is $M a=1.40, \mathrm{~T}_{\mathrm{s}, \mathrm{j}} / \mathrm{T}_{\infty}=$ 2.00, $M_{\text {flight }}=0.1$. 



Figure 20 Same as Figure 19 except data from present tests and flow condition is $M a=1.33, \mathrm{~T}_{\mathrm{s}, \mathrm{j}} / \mathrm{T}_{\infty}=$ 1.76, $M_{\text {flight }}=0.1$.

One other finding from the TWN10 test that requires looking into, partly for confirmation of repeatability and partly because it will be needed for other comparisons, is the impact of a second jet when the flows are unheated. Data from two nozzles in the presence of a surface was only acquired on unheated flows; hence the need to examine the case of unheated jets without a surface. This is especially important because a major finding from previous testing was that the impact of a second unheated jet was not as strong as when the jets were heated.

Figure 21 uses the same format as previous figures to present the impact of a second jet at $M a=0.9$ when the jets are unheated. Comparing Figure 21 with Figure 18, the impact on the observer at $\phi=90^{\circ}$ was not radically different, but adding the second jet did add $\sim 1 \mathrm{~dB}$ more noise when the jets were unheated than when they were heated. To the $\phi=0^{\circ}$ observer, however, the second jet added $\sim 1 \mathrm{~dB}$ less when the jets were unheated. So while the impact of adding a second jet was not axisymmetric in either case, it was significantly less asymmetric when the jets were cold than when they were heated. If the second heated jet produced a $6 \mathrm{~dB}$ asymmetry at the aft angles, the second unheated jet produced only a $4 \mathrm{~dB}$ asymmetry, and not over as broad a range of angles.
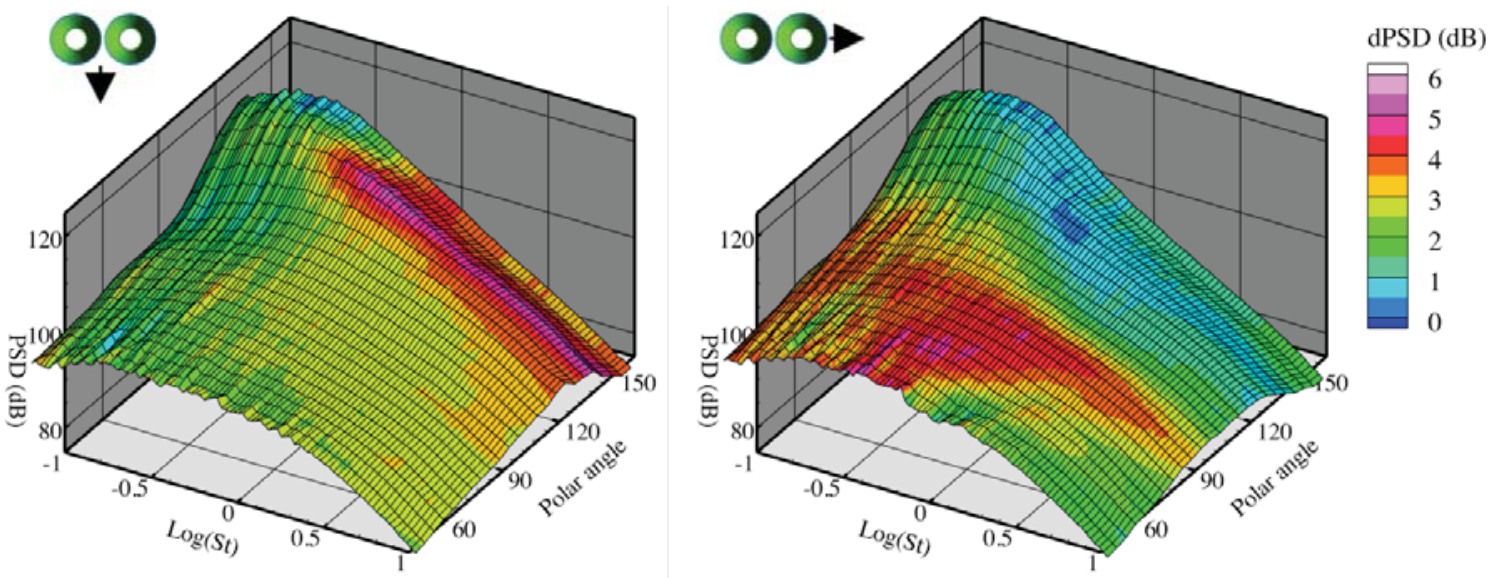

Figure 21 Difference in spectral directivity caused by second round jet with spacing $s / D=2.62$ as seen by observer normal to their common plane $\left(\phi=0^{\circ}\right.$; left) and in line with jets $\left(\phi=90^{\circ}\right.$; right). Shape is spectral directivity of two jets. Flow condition is unheated $M a=0.9, M_{\text {flight }}=0$. 


\section{G. Objective 7: Determine the impact of jet aspect ratio on the acoustic spectral directivity from twin rectangular jets.}

The twin jet effect is predominantly characterized by a change in directivity in the azimuthal direction at aft angles. As shown in Figure 22 and Figure 23, and compared against Figure 19 and Figure 20, the characteristics of the twin jet effect were similar with either round or rectangular twin jets. The 8:1 rectangular nozzles with hot flow showed a slightly stronger impact at $\phi=90^{\circ}$, and a slighter milder one at $\phi=0^{\circ}$.
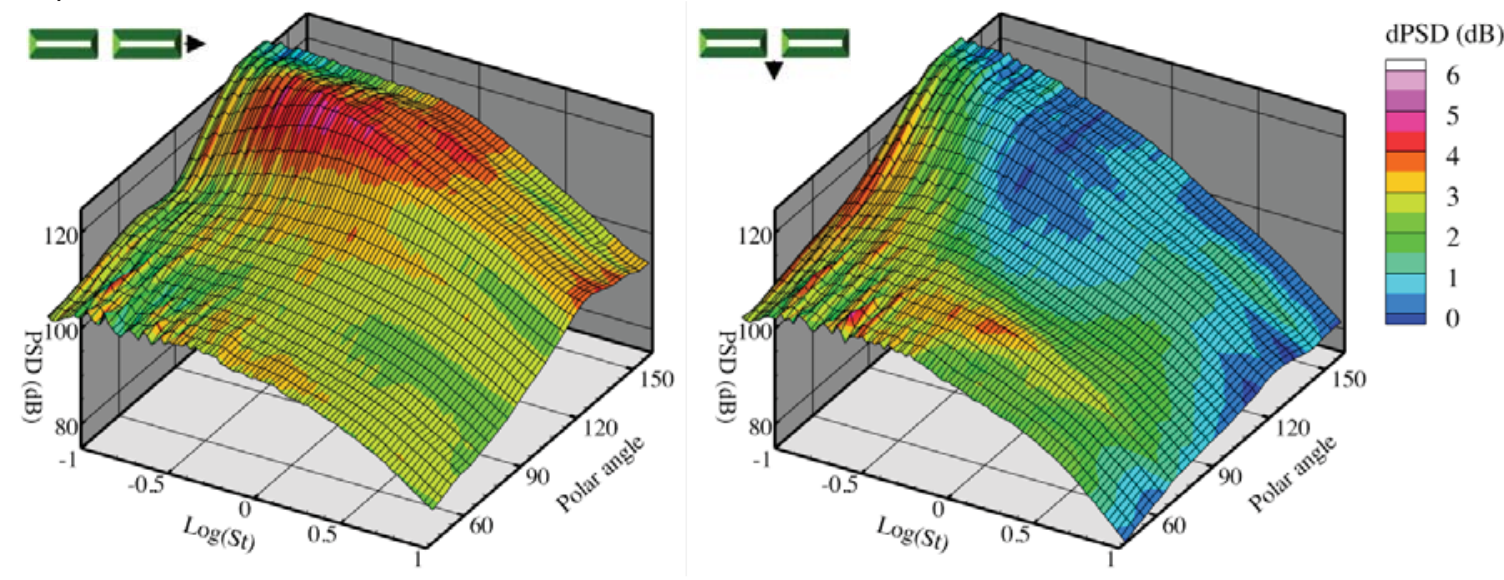

Figure 22 Difference in spectral directivity caused by adding second 8:1 rectangular jet to a single 8:1 rectangular jet with spacing $s / D=3.45$ as seen by observer normal to their common plane $\left(\phi=0^{\circ}\right.$; left $)$ and in line with jets $\left(\phi=90^{\circ}\right.$; right). Shape is spectral directivity of two jets. Flow condition is $M a=$ 1.33, $\mathrm{T}_{\mathrm{s}, \mathrm{j}} / \mathrm{T}_{\infty}=1.76, M_{\text {flight }}=0$. Compare with Figure 19.
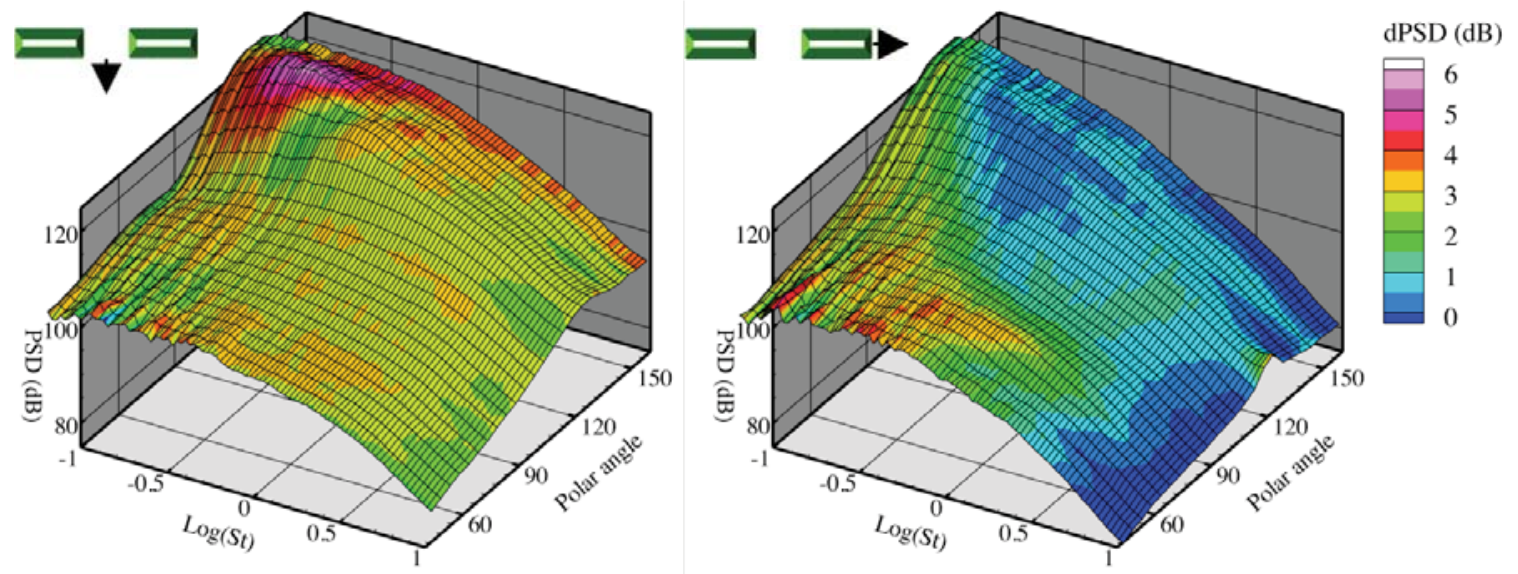

Figure 23 Same as Figure 22 except spacing $s / D=5.26$. Compare with Figure 20.

\section{H. Objective 8: Determine the impact of nearby surface on the acoustic spectral directivity from twin round jets.}

To add another degree of complexity to the installation problem, this section considers how the effect of adding a second jet was changed when a surface was coincident with the nozzle lip line. In the figures below, the color contours denote the difference in noise, two nozzles minus one nozzle. Figure 24 acts as a baseline case, presenting the difference for isolated nozzles, no surface. Figure 25 -Figure 27 present the same calculation when surfaces of different length $x_{T E}$ were present with surfaces flush with the nozzle lips, $h=0$. 

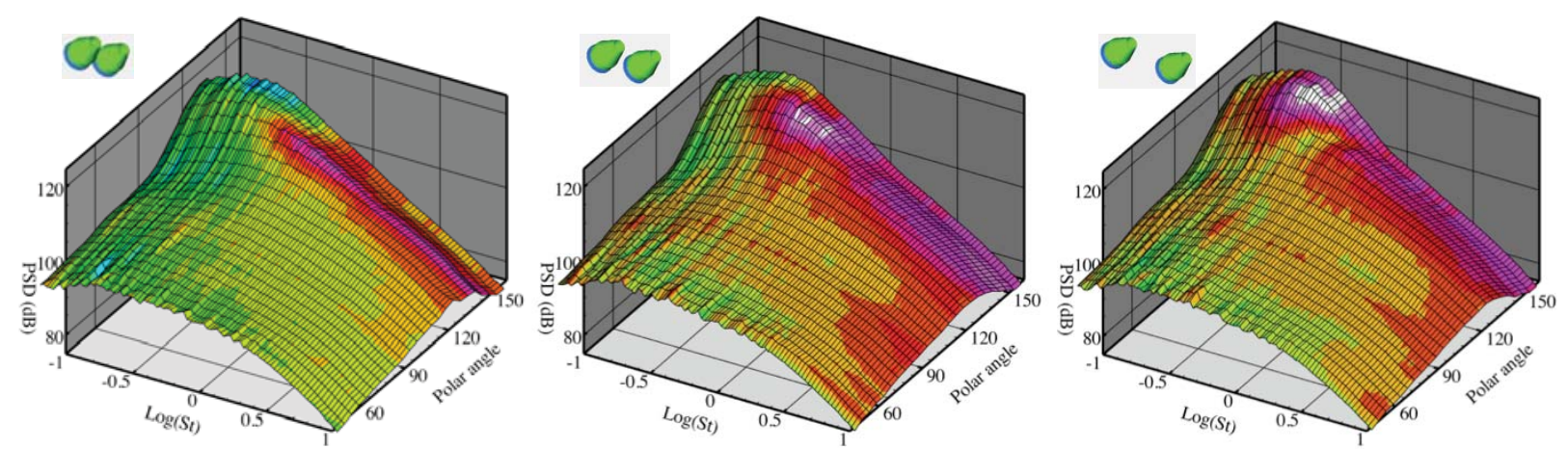

Figure 24 Difference in spectral directivity caused by adding a second round jet in isolation (no surface). Plots are for different spacings (left to right: $s / D=2.62,4.12,5.62$ ). Flow condition is unheated $M a=$ $0.9, M_{\text {flight }}=0$. Observer is in $\phi=0^{\circ}$ plane (normal to plane containing jet axes). Color scale spans from 0 to $6 d B$ as in Figure 23.

As shown in Figure 24 the impact of adding a second jet became stronger to the observer at $\phi=0^{\circ}$ the further apart the two jets were. When they were $s / D=2.62$ apart the second jet added roughly $3 \mathrm{~dB}$ to most angles and frequencies, a few $\mathrm{dB}$ more in the aft polar angles at high frequency. As the separation increases to $s / D=4.12$, the second jet added more than $3 \mathrm{~dB}$ generally, and by $s / D=5.62$ the second jet added $5-6 \mathrm{~dB}$ in the aft angles, including the peak of the noise directivity.

Now consider the change in noise caused by adding a second jet when a surface is present. In data presentations similar to Figure 24 the color contours will show the difference in noise of two jets with a surface minus a single jet with a surface. Figure 25 shows the result for a short surface $\left(x_{T E} / D=1.35\right)$, where the second jet had roughly the same effect (within $0.5 \mathrm{~dB}$ ) as the isolated jet for spacings $s / D=2.62$ and 4.12. At the largest separation $(s / D=5.62)$ the second jet added less noise (by roughly $1 \mathrm{~dB}$ ) than if the surface was not present.

When the surface was slightly longer $\left(x_{T E} / D=2\right.$; Figure 26$)$ the increase in noise from adding a second jet was slightly higher $(0.5 \mathrm{~dB})$ than without the surface for the close spacing of jets, roughly the same for the medium and large spacings. The ripples in the shape of the spectral directivity are indicative of more complicated effects caused by the surface, but these effects were unaffected by the addition of the second jet as the constant color contours indicate.

What was most dramatic was what happened when the surface was made even longer $\left(x_{T E} / D=6\right.$; Figure 27). From the change in shape of the spectral directivity between plots in Figure 26 and Figure 27 it is clear that with the longer surface the jets produced much greater noise than with the shorter surface. Moreover, while the addition of a second jet produced almost the same effect as when there was not a surface for the closely spaced jets, the addition of a second jet when the spacing is $s / D=4.12$ produced dramatically more noise than the expected $3 \mathrm{~dB}$ from source doubling. The complicated spectral character shown in the shape of the spectral directivity also alludes to more complex behavior, suggesting a closer look at this case in the future. 

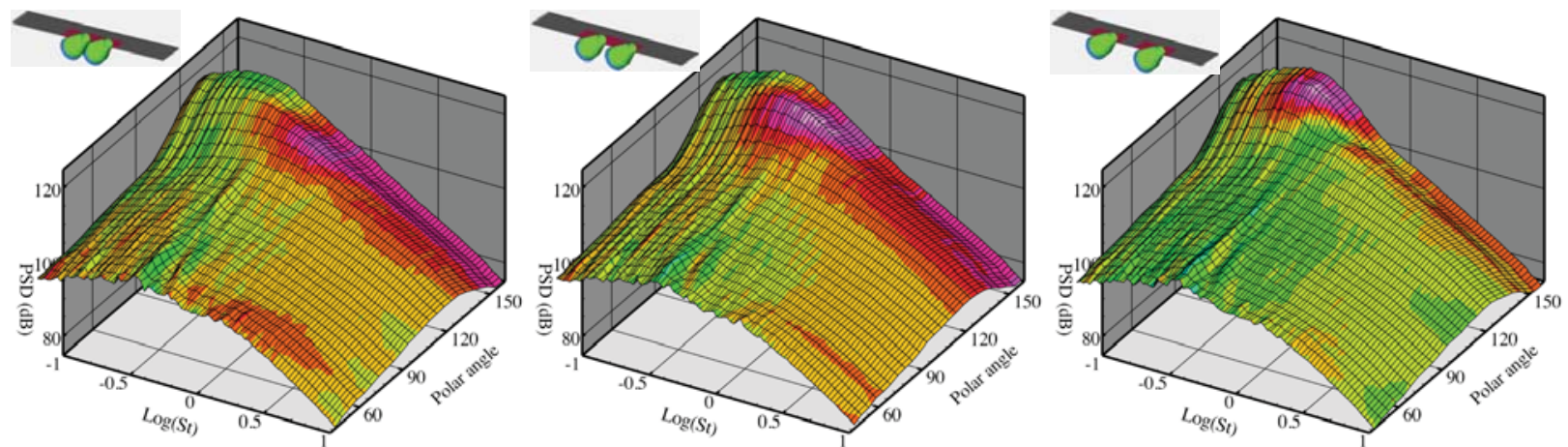

Figure 25 Same as Figure 24 except nozzles installed under the $x_{T E}=2.7$ " surface with zero standoff $(h=$ $0)$ in 'reflecting' configuration to microphones $\left(\phi=0^{\circ}\right)$.
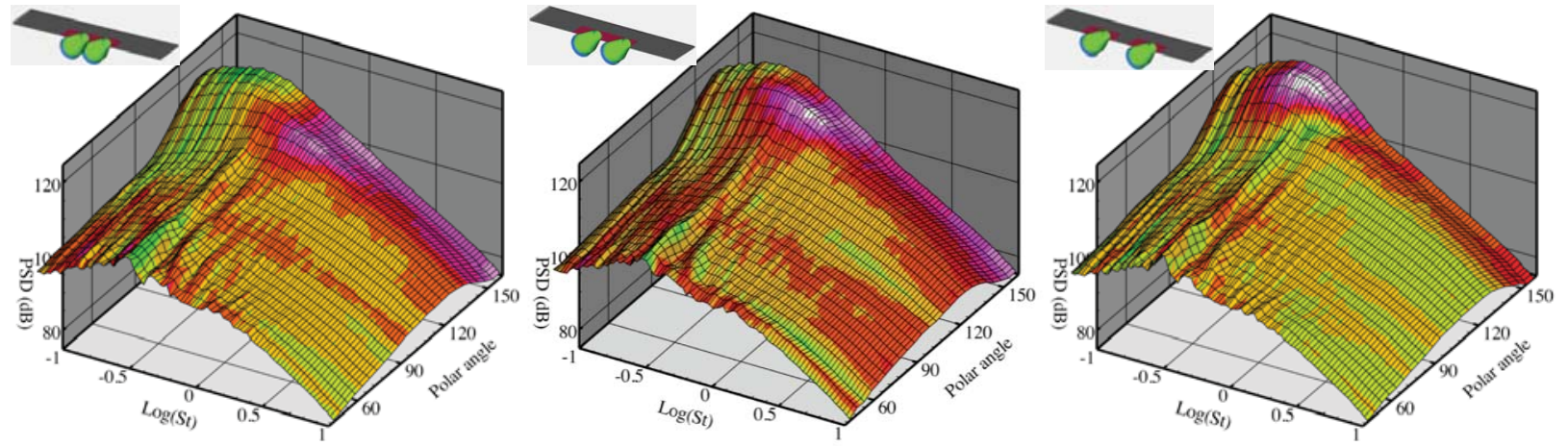

Figure 26 Same as Figure 25 except $x_{T E}=4$ ".
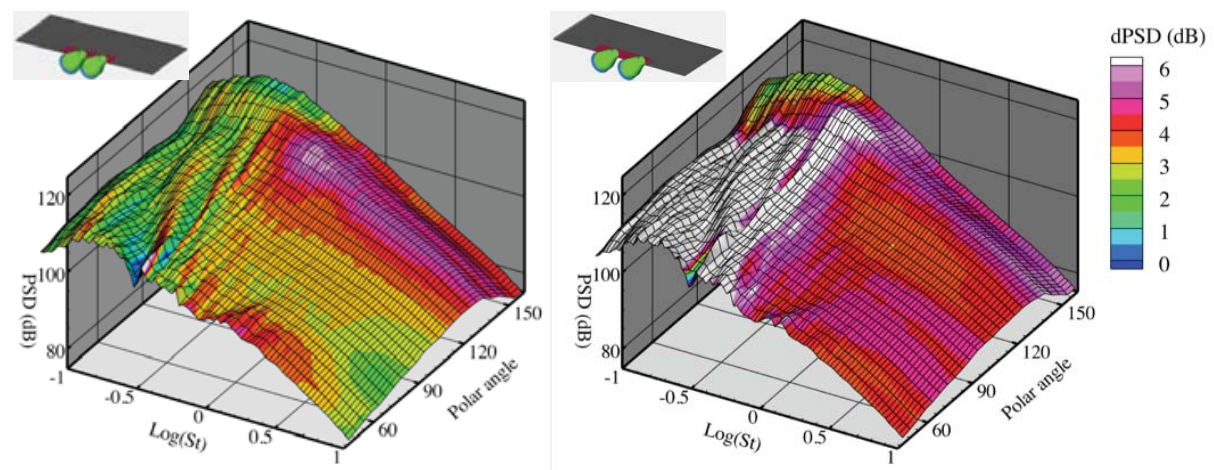

Figure 27 Same as Figure 25 except $x_{T E}=12$ ”. No data for $s / D=5.62$.

\section{Objective 9: Determine the impact of nearby surface on the acoustic spectral directivity from twin rectangular jets.}

Finally, consider the case that was shown diametrically opposite the simple single round isolated jet in Figure 1. Here, all the effects of aspect ratio, surface, and spacing are considered together. As in the case of the round jets in the section just above, the presentation starts (Figure 28) with the case of isolated twin nozzles, this time of aspect ratio 8:1. Only two spacings, $s / D_{e}=3.45$ and 5.25, are shown because the two nozzles would overlap if installation at $s / D_{e}=2.45$ was attempted! The shape of the spectral directivity surface was significantly different than in the case of round jets (Figure 24). The difference between one and two jets was similar in distribution but reduced by about $1 \mathrm{~dB}$ from the same geometry with round nozzles. 

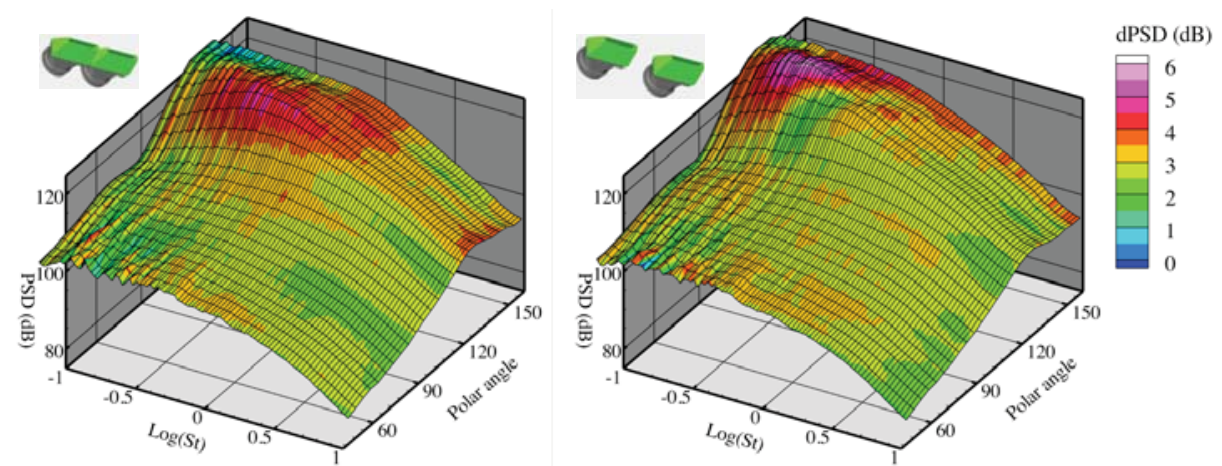

Figure 28 Difference in spectral directivity caused by adding a second rectangular (A8Z0) jet in isolation (no surface). Plots are for different spacings $\left(s / D_{e}=3.45,5.25\right)$. Flow condition is unheated $M a=0.9$, $M_{\text {flight }}=0$. Observer is in $\phi=0^{\circ}$ plane (normal to plane containing jet axes). Color scale spans from 0 to $6 d B$.

The impact of adding the second jet changed dramatically, however, as soon as a surface was introduced. Looking at Figure 29 and Figure 30 it is observed that the plots for the wider spacing $\left(s / D_{e}=\right.$ 5.25 ) show a difference in the sound of one and of two jets that is within $\pm 1 \mathrm{~dB}$ of the difference in the sound when the jets were isolated. The difference in color contours, with and without the surface, is significant, but nothing compared with the difference when the jets were more closely spacing $\left(s / D_{e}=\right.$ 3.45). The impact of adding a second rectangular nozzle at the closest spacing was quite dramatic for both short and long surface lengths. From the shape of the spectral directivity surface in Figure 29 (two rectangular nozzles with surface) compared with the shape of the spectral directivity surface in Figure 28 (two rectangular nozzles without surface), it is clear that the surface adds dramatically to the high frequency noise in the case of the 8:1 rectangular nozzle. Moreover, this strong change in high frequency noise produced by adding the surface only occurred because of the presence of the second rectangular nozzle at the close spacing. Clearly, there were significant changes to the flow and to the noise generation when one combined high aspect ratio nozzles with extended surfaces.
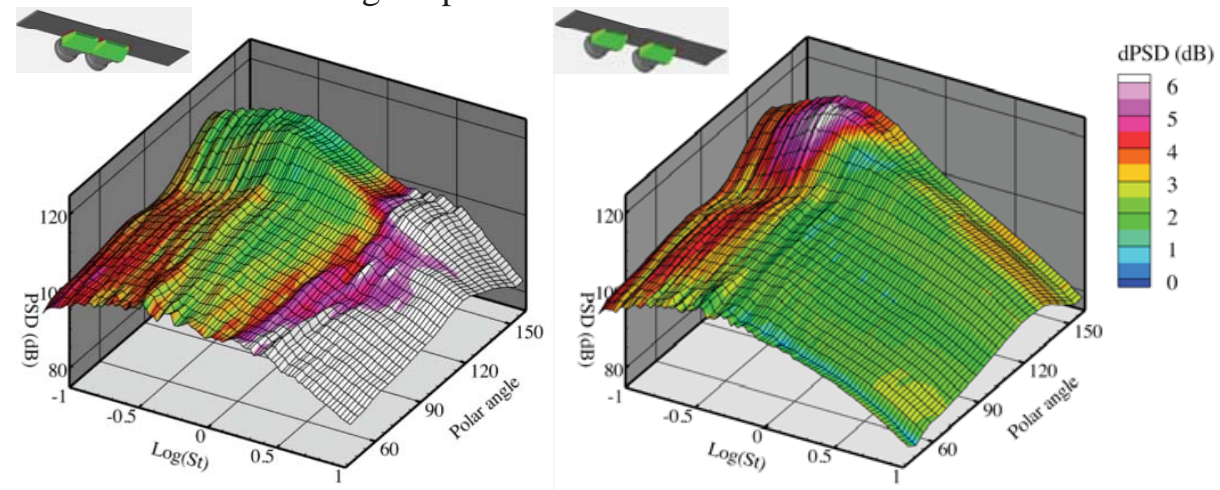

Figure 29 Same as Figure 28 except nozzles installed under the $x_{T E}=2.7$ " surface with zero standoff $(h=$ $0)$ in 'reflecting' configuration to microphones $\left(\phi=0^{\circ}\right)$ 

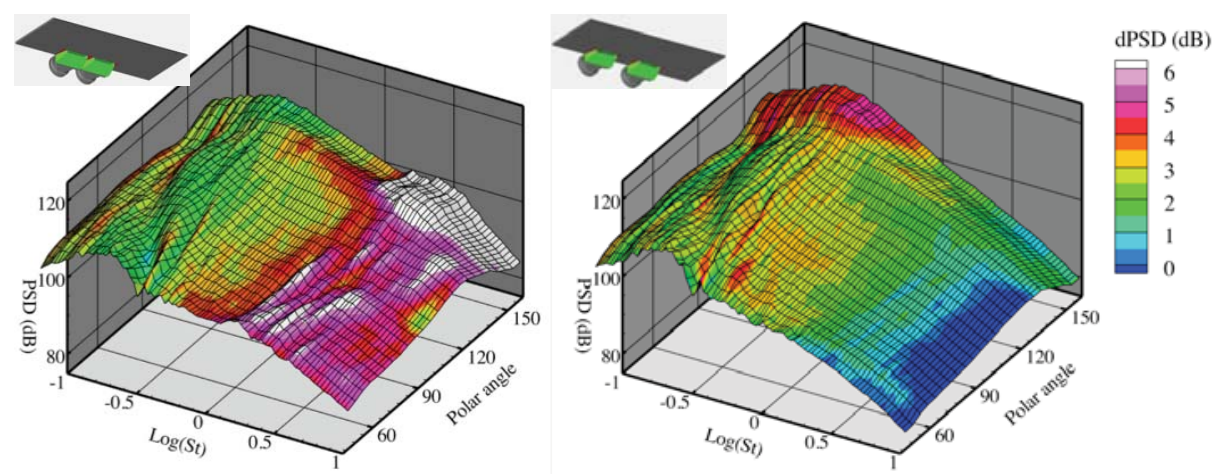

Figure 30 Same as Figure 29 except $x_{T E}=12 ”$.

\section{Discussion}

Having established the uncertainty limits of the data collected by checks on self-consistency and repeatability in Objectives 1-3 and 6, and having briefly reviewed the individual effects of surface, pairing, and aspect ratio in Objectives 4,5 and 7-9, we can look ahead at the task of creating empirical models of each effect and the likelihood of success in having each effect (spacing, surface parameters, aspect ratio, flight effect) be independent from each other.

From the presentation of Objective 4 it appears that aspect ratio may not be a strong factor when considering the impact of the surface on the noise. Partly this is because the acoustic impact of the surface is very large, up to $15 \mathrm{~dB}$ or more. However, this dramatic impact is largely confined to frequencies below $\mathrm{St}=1$. If one wants to capture the impact of the surface at frequencies above $\mathrm{St}=1$, which would be where the noise generally impacts human hearing in practical commercial aircraft, then the acoustic impact of the surface is not as strong and the effect of aspect ratio will need to be accounted for.

Similarly, from the presentation of Objective 5, aspect ratio plays a small role on the reduction of noise by flight. The difference in flight effect caused by aspect ratio is mostly limited to the peak aft region where the benefit is reduced with increase in aspect ratio.

From data presented in Objective 6 the modeling for the impact of adding a second jet will need to account for jet temperature. Otherwise, the impact is only slightly sensitive to the jet spacing for round jets. When the jets are not round, data from Objective 7 shows that the same holds true for jets of different aspect ratio; although there is a measureable impact of aspect ratio on the effect of adding a second jet and its sensitivity to spacing, this impact is less than $1 \mathrm{~dB}$ different than the round jet.

Once surfaces get involved the smooth change in noise with geometric parameter is lost. From Objective 8 above, the impact of adding a second round jet when the nozzle is close to a surface is relatively benign for short surfaces. The effect of adding a second round jet is greater when a surface is present than when no surface is present for close spacings; the effect of adding a second round jet is less when the jets are separated by more than 5 jet diameters. However, the case of a long surface $\left(x_{T E} / D=6\right)$ with moderately spaced jets $(s / D=4.12)$ shows an extreme increase in low frequencies at all angles and an addition of more than $4 \mathrm{~dB}$ at all angles and frequencies when the second round jet was added. The results became even more complicated when the nozzles were of high aspect ratio. As shown the section on Objective 9, strange, noise-increasing effects were noted when a second rectangular nozzle was added closely spaced to the first with both short and long surfaces. These observations cast doubt on whether the addition of acoustic effects from doubling jets, changing aspect ratio, and adding surfaces will be successful in the presence of surfaces due to a complete change in the flow and its noise generation mechanisms.

Finally, it is noteworthy, that in no case was there a substantial benefit noted when increasing aspect ratio. Beneficial effects of flight on noise were reduced by increasing aspect ratio, jet-by-jet shielding was 
not improved by increasing aspect ratio, and the effect of mounting the nozzle near a surface, at least in the underwing position, was not favorable.

\section{Summary}

An ambitious test program has been completed, with the goals of providing a flow and acoustic database for highly integrated exhaust systems. The effects of high aspect ratio, close proximity of the plume with the airframe surface, and multiple exhaust nozzles have been assessed, both independently and in various combinations. This paper outlines the equipment and methods used, some of the checks made to assure that the data is accurate, and a sample look at data that supports several of the major objectives. Agreement between the two rigs that were used in the testing was shown to be within $0.5 \mathrm{~dB}$ for most frequencies and polar angles. Even though the hardware embodiment of the surface was somewhat different on the two rigs, the impact of the surface was measured to be the same to within $1 \mathrm{~dB}$ (out of impact of $15 \mathrm{~dB}$ ). Initial impressions are that nozzle aspect ratio is not a significant factor in jet by jet shielding and but is a significant factor in the noise generated when in the presence of a solid surface near the jet plume. Jet by jet shielding is much more significant for hot jets than for unheated ones, with significant reductions in noise in the plane of the two plumes but significant increases for most other azimuthal angles.

\section{Acknowledgements}

This research was carried out with support of the High Speed Project under NASA's Fundamental Aeronautics Program.

\section{References}

\footnotetext{
${ }^{1}$ Coles, W. D. "Jet-Engine Exhaust Noise from Slot Nozzles," NASA Technical Note D-60, Lewis Research Center, September 1959.

${ }^{2}$ Dorsch, R. G., E. A. Kresja and W. A. Olsen. "Blown Flap Noise Research," AIAA Paper No. 71-745, June 1971.

${ }^{3}$ Schrecker, G.O.H., "Turbulence and Aerodynamic Noise Characteristics of Jet Flap Type Exhaust Flows," Ph.D. Thesis, The University of Tennessee, Ph.D., 1972.

${ }^{4}$ Gruschka, H. D.; Schrecker, G. O., "Aeroacoustic characteristics of jet flap type exhausts," AIAA Paper 72-130, 1972.

${ }^{5}$ Olsen, W.A. and Boldman, D. "Preliminary Study of the Effect of the Turbulent Flow Field Around Complex Surfaces On Their Acoustic Characteristics," AIAA paper 1978-1123, presented at 11th Fluid and Plasma Dynamics Conference, Seattle, WA, July 10-12, 1978.

${ }^{6}$ Goldstein, M.E., Afsar, M.Z. and Leib, S.J. "Non-homogeneous rapid distortion theory on transversely sheared mean flows," J. Fluid Mechanics, 736, December 2013, pp 532 - 569.

${ }^{7}$ Brown, C.A. , "Jet-Surface Interaction Test: Far-Field Noise Results", J. Eng. Gas Turbines Power, 135(7), Jun. 2013.

${ }^{8}$ Erwin, J.P., Sinha, N. and Rodebaugh, G.P. "Noise predictions of a hot twin-jet impinging on a jet blast deflector," AIAA Paper 2013-0324, 51st AIAA Aerospace Sciences Meeting, Grapevine, TX, 07-10 January 2013.

${ }^{9}$ Munro, S.E. and Ahuja, K.K. "Development of a Prediction Scheme for High Aspect-Ratio Jet Noise," AIAA paper 2003-3255, presented at 9th AIAA/CEAS Aeroacoustics Conference, Hilton Head, South Carolina, 12-14 May 2003.

${ }^{10}$ Bridges, J. “Acoustic Measurements of Rectangular Nozzles with Bevel," AIAA Paper 2012-2252, presented at 18th AIAA/CEAS Aeroacoustics Conference, Colorado Springs, CO, 5 June 2012.
} 
${ }^{11}$ Bridges, J "Noise from Aft Deck Exhaust Nozzles_-Differences in Experimental Embodiments," AIAA Paper 2014-0876, presented at SciTech 2014, Nat'l Harbor, MD, January 2014.

${ }^{12}$ Bridges, J. and Brown, C.A., "Validation of the Small Hot Jet Acoustic Rig for Jet Noise Research," AIAA Paper 2005-2846, presented at $11^{\text {th }}$ AIAA/CEAS Aeroacoustics Conference, Monterrey, California, June 2005.

${ }^{13}$ Brown, C.A. , "Jet-Surface Interaction Test: Far-Field Noise Results", J. Eng. Gas Turbines Power, 135(7), Jun. 2013.

${ }^{14}$ Bozak, R, and Henderson, B.S. “Aeroacoustic Experiments with Twin Jets,” AIAA Paper 2011-2790, $17^{\text {th }}$ AIAA/CEAS Aeroacoustics Conference, Portland, OR, 08 June 2011.

${ }^{15}$ Frate, F.C. and Bridges, J., "Extensible Rectangular Nozzle Model System," AIAA Paper 2011-0975, Orlando, FL, January 2011.

${ }^{16}$ Plumblee, H.E., Burrin, R.H., Lau, J.C., Morfey, C.L. Morris, P.J., Smith, D.M., Tanna, H.K., Tester, B.J., Whiffen, M.C., "The generation and radiation of supersonic jet noise, part III, Turbulent mixing noise data," AFAPL-TR-76-45, 1976.

${ }^{17}$ Zaman, K., Fagan, A., Clem, M.C. and Brown, C.A. "Resonant Interaction of a Rectangular Jet with a Flatplate," AIAA Paper 2014-0877, presented at SciTech 2014, Nat'1 Harbor, MD, January 2014. 